\title{
Twin Positive Solutions for Schrödinger-Kirchhoff-Type Problem with Singularity and Critical Exponents
}

\author{
Wei Han (iD) and Yangyang Zhao \\ Department of Mathematics, North University of China, Taiyuan, Shanxi 030051, China \\ Correspondence should be addressed to Wei Han; sh_hanweiweil@126.com
}

Received 27 January 2018; Accepted 25 March 2018; Published 2 May 2018

Academic Editor: Dumitru Motreanu

Copyright (c) 2018 Wei Han and Yangyang Zhao. This is an open access article distributed under the Creative Commons Attribution License, which permits unrestricted use, distribution, and reproduction in any medium, provided the original work is properly cited.

We study in this paper the following singular Schrödinger-Kirchhoff-type problem with critical exponent $-\left(a+b \int_{\Omega}|\nabla u|^{2} d x\right) \Delta u+$ $u=Q(x) u^{5}+\mu|x|^{\alpha-2} u+f(x)\left(\lambda / u^{\gamma}\right)$ in $\Omega, u=0$ on $\partial \Omega$, where $a, b>0$ are constants, $\Omega \subset R^{3}$ is a smooth bounded domain, $0<\alpha<1, \lambda>0$ is a real parameter, $\gamma \in(0,1)$ is a constant, and $0<\mu<a \mu_{1}\left(\mu_{1}\right.$ is the first eigenvalue of $-\Delta u=\mu|x|^{\alpha-2} u$, under Dirichlet boundary condition). Under appropriate assumptions on $Q$ and $f$, we obtain two positive solutions via the variational and perturbation methods.

\section{Introduction and Main Result}

In this paper, we consider the following SchrödingerKirchhoff-type problem with Dirichlet boundary value conditions

$$
\begin{aligned}
& -\left(a+b \int_{\Omega}|\nabla u|^{2} d x\right) \Delta u+u \\
& \quad=Q(x) u^{5}+\mu|x|^{\alpha-2} u+f(x) \frac{\lambda}{u^{\gamma}} \quad \text { in } \Omega, \\
& u=0 \quad \text { on } \partial \Omega,
\end{aligned}
$$

where $\Omega$ is a smooth bounded domain in $\mathbb{R}^{3}, 0<\alpha<1$, $\lambda>0$ is a real parameter, $\gamma \in(0,1)$ is a constant, and $0<$ $\mu<a \mu_{1}\left(\mu_{1}\right.$ is the first eigenvalue of $-\Delta u=\mu|x|^{\alpha-2} u$, under Dirichlet boundary condition), and coefficient functions $Q \epsilon$ $C(\bar{\Omega}), f \in C(\bar{\Omega})$ are positive, which have at least one minimum. This kind of problem has been widely concerned. The Schrödinger-Kirchhoff-type problem in the effect of electromagnetic field on current motion in physics and the basic law of physical world material movement has been widely used. In recent years, the following elliptic problems have been extensively studied by many researchers:

$$
\begin{aligned}
-\left(a+b \int_{\mathbb{R}^{N}}|\nabla u|^{2} d x\right) \Delta u+V(x) u= & f(x, u), \\
& u \in H^{1}\left(\mathbb{R}^{N}\right),
\end{aligned}
$$

where $V: \mathbb{R}^{N} \rightarrow \mathbb{R}, f \in C\left(\mathbb{R}^{N} \times \mathbb{R}, \mathbb{R}\right), N=1,2,3$, and $a, b>0$ are constants. In [1], Li and Ye considered the existence of positive ground state solutions to the following Kirchhoff-type problem with pure power nonlinearities:

$$
\begin{aligned}
& -\left(a+b \int_{\mathbb{R}^{3}}|\nabla u|^{2} d x\right) \Delta u+V(x) u=|u|^{p-1} u \\
& x \in \mathbb{R}^{3} \text {, } \\
& u \in H^{1}\left(\mathbb{R}^{3}\right) \text {, } \\
& u>0 x \in \mathbb{R}^{3} .
\end{aligned}
$$

Furthermore, Chen and Tang [2] studied the following Kirchhoff-type equation:

$$
\begin{aligned}
&-\left(a+b \int_{\mathbb{R}^{3}}|\nabla u|^{2} d x\right) \Delta u+V(x) u=f(x, u) \\
& \\
& x \in \mathbb{R}^{3}, \\
& u(x) \longrightarrow 0 \\
& \quad|x| \longrightarrow \infty,
\end{aligned}
$$

and their result unifies both asymptotically cubic and supercubic cases, which generalizes and improves the existing 
ones. In [3], Huy and Quan investigated the equation $-M(x,\|u\|) \Delta_{p} u=\lambda f(x, u, \nabla u)-g(x, u, \nabla u)$ in $\Omega, u=0$ on $\partial \Omega$. They proved the existence results for both nondegenerate and degenerate cases of the function $M$ and combined the fixed point index theory with the cone theoretic argument which are key technical ingredients to obtain the main results. In [4], Figueiredo and Severo established the existence of a positive ground state solution for a Kirchhoff problem in $\mathbb{R}^{2}$ involving critical exponential growth. Moreover, Ricceri [5] obtained some results of a totally new type about a class of nonlocal problems. In [6], the authors studied the multiplicity of positive solutions for a class of Kirchhoff type of equations with the nonlinearity containing both singularity and critical exponents:

$$
\begin{aligned}
-\left(a+b \int_{\Omega}|\nabla u|^{2} d x\right) \Delta u & =u^{5}+\frac{\lambda}{u^{\gamma}} \quad \text { in } \Omega, \\
u & =0 \quad \text { on } \partial \Omega .
\end{aligned}
$$

In the case that $V(x) \equiv 0$ in (2), it is related to the stationary analogue of the equation

$$
\rho \frac{\partial^{2} u}{\partial t^{2}}-\left(\frac{P_{0}}{h}+\frac{E}{2 L} \int_{0}^{L}\left|\frac{\partial u}{\partial x}\right|^{2} d x\right) \frac{\partial^{2} u}{\partial x^{2}}=0,
$$

presented by Kirchhoff in [7]. In [8], Lions introduced an abstract functional analysis framework to the following equation:

$$
u_{t t}-\left(a+b \int_{\Omega}|\nabla u|^{2} d x\right) \Delta u=f(x, u)
$$

proposed by $\mathrm{He}$ and Zou in [9] as an existence of the classical D'Alembert's wave equations for free vibration of elastic strings. After the vanguard work of Lions [8], where a functional analysis method was presented, the Kirchhofftype equations started to arouse attention of researchers. D'Ancona and Spagnolo [10] studied the existence of a global classical periodic solution with real analytic data. Arosio and Panizzi [11] proved the well-posedness of the Cauchy problem concerned (7) in the Hadamard sense as a special case of an abstract second-order Cauchy problem in a Hilbert space. Perera and Zhang [12] obtained a nontrivial solution via Yang index and critical group. Caponi and Pucci [13] proved existence, multiplicity, and asymptotic behavior of entire solutions for a series of stationary Kirchhoff fractional $p$-Laplacian equations. In [14-16], the authors proved the existence of a nontrivial solution without the AmbrosettiRabinowitz condition. Moreover, we found in [17-19] that the authors considered the existence and multiplicity of solutions by Nehari manifold. In [20], Shen and Yao obtained two positive solutions about the following Kirchhoff problem:

$$
\begin{aligned}
& -\left(a+b \int_{\mathbb{R}^{3}}|\nabla u|^{2} d x\right) \Delta u+u \\
& \quad=Q(x)|u|^{4} u+\lambda f(x)|u|^{q-2} u \text { in } \mathbb{R}^{3}, \\
& u \in H^{1}\left(\mathbb{R}^{3}\right),
\end{aligned}
$$

under some assumptions on the weight functions $Q(x)$ and $f(x)$. The related developments of the existence of solutions for the Kirchhoff-type problems also can be found in [21-24].

However, up till now, no paper has appeared in the literature which discusses the singular Schrödinger-Kirchhofftype problem with critical exponent. This paper attempts to fill in this gap in the literature; in this paper, we obtain the multiple positive solutions for the problem via the variational and perturbation methods. Throughout this paper, we make use of the following notations:

(1) The norm in $H_{0}^{1}(\Omega)$ is equipped with the norm $\|u\|^{2}=$ $\int_{\Omega}\left(a|\nabla u|^{2}+|u|^{2}\right) d x, \forall u \in H_{0}^{1}(\Omega)$, and the norm in $L^{p}(\Omega)$ is denoted by $|\cdot|_{p}$.

(2) $u_{n}^{+}(x)=\max \left\{u_{n}(x), 0\right\}, u_{n}^{-}(x)=\max \left\{-u_{n}(x), 0\right\}$.

(3) $C_{1}, C_{2}$, and $C_{3}$ denote various positive constants, which may vary from line to line.

(4) We denote by $S_{r}$ (resp., $B_{r}$ ) the sphere (resp., the closed ball) of center zero and radius $r$; that is, $S_{r}=\left\{u \in H_{0}^{1}(\Omega)\right.$ : $\|u\|=r\}, B_{r}=\left\{u \in H_{0}^{1}(\Omega):\|u\| \leq r\right\}$. Let $S$ be the Sobolev constant; namely,

$$
S:=\inf _{u \in H_{0}^{1}(\Omega) \backslash\{0\}} \frac{\int_{\Omega}|\nabla u|^{2} d x}{\left(\int_{\Omega}|u|^{6} d x\right)^{1 / 3}} .
$$

We define the functional

$$
\begin{aligned}
I(u)= & \frac{1}{2} \int_{\Omega}\left(a|\nabla u|^{2}+|u|^{2}\right) d x+\frac{b}{4}\left(\int_{\Omega}|\nabla u|^{2} d x\right)^{2} \\
& -\frac{\mu}{2} \int_{\Omega}|x|^{\alpha-2}|u|^{2} d x-\frac{1}{6} \int_{\Omega} Q(x)\left|u^{+}\right|^{6} d x \\
& -\frac{\lambda}{1-\gamma} \int_{\Omega} f(x)\left|u^{+}\right|^{1-\gamma} d x .
\end{aligned}
$$

In general, a function $u$ is called a weak solution of (1) if $u \in$ $H_{0}^{1}(\Omega)$, and for all $v \in H_{0}^{1}(\Omega)$, it holds that

$$
\begin{gathered}
\int_{\Omega}(a \nabla u \nabla v+u v) d x+b \int_{\Omega}|\nabla u|^{2} d x \int_{\Omega} \nabla u \nabla v d x \\
-\mu \int_{\Omega}|x|^{\alpha-2} u v d x-\int_{\Omega} Q(x)\left|u^{+}\right|^{5} v d x \\
-\lambda \int_{\Omega} f(x)\left|u^{+}\right|^{-\gamma} v d x=0,
\end{gathered}
$$

and for $\alpha>0$, we consider the following perturbed problem:

$$
\begin{aligned}
& -\left(a+b \int_{\Omega}|\nabla u|^{2} d x\right) \Delta u+u \\
& =Q(x)\left|u^{+}\right|^{5}+\mu|x|^{\alpha-2} u+\frac{\lambda f(x)}{\left(u^{+}+\alpha\right)^{\gamma}} \text { in } \Omega, \\
& u=0 \text { on } \partial \Omega .
\end{aligned}
$$


Solution (12) corresponds to critical point of $C^{1}$-functional on $H_{0}^{1}(\Omega)$ by

$$
\begin{aligned}
I_{\alpha}(u)= & \frac{1}{2} \int_{\Omega}\left(a|\nabla u|^{2}+|u|^{2}\right) d x+\frac{b}{4}\left(\int_{\Omega}|\nabla u|^{2} d x\right)^{2} \\
& -\frac{\mu}{2} \int_{\Omega}|x|^{\alpha-2}|u|^{2} d x-\frac{1}{6} \int_{\Omega} Q(x)\left|u^{+}\right|^{6} d x \\
& -\frac{\lambda}{1-\gamma} \int_{\Omega} f(x)\left(\left(u^{+}+\alpha\right)^{1-\gamma}-\alpha^{1-\gamma}\right) d x .
\end{aligned}
$$

The main result can be described as follows.

Theorem 1. Assume that $\gamma \in(0,1), a>0, b \geq 0$, and $Q \in$ $C(\bar{\Omega}), f \in C(\bar{\Omega})$ are positive, which have at least one minimum. Then, there exists $\lambda_{*}>0$, such that, for any $\lambda \in\left(0, \lambda_{*}\right)$, problem (1) has at least two different positive solutions.

This paper is organized as follows. In Sections 2 and 4, we give the proof of Theorem 1. In Section 3, we consider the existence of at least one mountain-pass solution of the perturbation problem.

\section{Existence of a First Solution of Problem (1)}

In this section, our main work is to prove that problem (1) has a local minimum solution in $H_{0}^{1}(\Omega)$.

Lemma 2. There exist constants $\lambda_{0}>0$ and $R, \rho>0$ such that, for all $0<\lambda<\lambda_{0}$, one has

$$
\begin{gathered}
\left.I(u)\right|_{u \in S_{R}} \geq \rho, \\
\inf _{u \in B_{R}} I(u)<0 .
\end{gathered}
$$

Proof. By Hölder inequality and (9), we can obtain that

$$
\begin{aligned}
& \int_{\Omega} f(x)\left(u^{+}\right)^{1-\gamma} d x \leq \int_{\Omega} f(x)|u|^{1-\gamma} d x \leq \max _{x \in \bar{\Omega}} f(x) \\
& \cdot\left(\int_{\Omega}\left(|u|^{1-\gamma}\right)^{6 /(1-\gamma)} d x\right)^{(1-\gamma) / 6} \\
& \cdot\left(\int_{\Omega} 1^{6 /(5+\gamma)} d x\right)^{(5+\gamma) / 6} \leq \max _{x \in \bar{\Omega}} f(x)|u|_{6}^{1-\gamma} \\
& \cdot|\Omega|^{(5+\gamma) / 6} \leq|\Omega|^{(5+\gamma) / 6} S^{-(1-\gamma) / 2} \max _{x \in \bar{\Omega}} f(x) \\
& \cdot \int_{\Omega}|\nabla u|^{2} d x .
\end{aligned}
$$

Therefore, from (9) and (15), we have

$$
\begin{gathered}
I(u)=\frac{1}{2} \int_{\Omega}\left(a|\nabla u|^{2}+|u|^{2}\right) d x+\frac{b}{4}\left(\int_{\Omega}|\nabla u|^{2} d x\right)^{2} \\
-\frac{\mu}{2} \int_{\Omega}|x|^{\alpha-2}|u|^{2} d x-\frac{1}{6} \int_{\Omega} Q(x)\left|u^{+}\right|^{6} d x \\
-\frac{\lambda}{1-\gamma} \int_{\Omega} f(x)\left|u^{+}\right|^{1-\gamma} d x \geq \frac{1}{2}\left(a-\frac{\mu}{\mu_{1}}\right)
\end{gathered}
$$

$$
\begin{aligned}
& \cdot \int_{\Omega}|\nabla u|^{2} d x-\frac{1}{6} S^{-3} \int_{\Omega} Q(x)|\nabla u|^{6} d x \\
& -\frac{\lambda}{1-\gamma}|\Omega|^{(5+\gamma) / 6} S^{-(1-\gamma) / 2} \max _{x \in \bar{\Omega}} f(x) \int_{\Omega}|\nabla u|^{2} d x \\
& \geq \int_{\Omega}|\nabla u|^{1-\gamma} d x\left[\frac{1}{2}\left(a-\frac{\mu}{\mu_{1}}\right) \int_{\Omega}|\nabla u|^{1+\gamma} d x\right. \\
& -\frac{1}{6} S^{-3} \int_{\Omega} Q(x)|\nabla u|^{5+\gamma} d x \\
& \left.-\frac{\lambda}{1-\gamma}|\Omega|^{(5+\gamma) / 6} S^{-(1-\gamma) / 2} \int_{\Omega} f(x) d x\right] .
\end{aligned}
$$

We can set $g(t)=(1 / 2)\left(a-\mu / \mu_{1}\right) t^{1+\gamma}-(1 /$ 6) $S^{-3} t^{5+\gamma} \int_{\Omega} Q(x) d x$ for $t>0$; therefore, there exists a constant $R=\left(3\left(a \mu_{1}-\mu\right)(1+\gamma) S^{3} / \mu_{1}(5+\gamma) \int_{\Omega} Q(x) d x\right)^{1 / 4}>0$, such that $\max _{t>0} g(t)=g(R)>0$. We can let $\lambda_{0}=((1-$ $\left.\gamma) S^{(1-\gamma) / 2} /|\Omega|^{(5+\gamma) / 6} \int_{\Omega} f(x) d x\right) g(R)$; then, there exists a constant $\rho>0$ such that $\left.I(u)\right|_{S_{R}} \geq \rho$ for all $0<\lambda<\lambda_{0}$. For given $R$, we can deduce $u \in B_{R}$ with $u^{+} \neq 0$; one has

$$
\lim _{t \rightarrow 0} \frac{I(t u)}{t^{1-\gamma}}=-\frac{\lambda}{1-\gamma} \int_{\Omega} f(x)\left|u^{+}\right|^{1-\gamma} d x<0 .
$$

Hence, we have $I(t u)<0$ for all $u^{+} \neq 0$ and $t$ small enough. So, for $\int_{\Omega} d x$ sufficiently small, we have

$$
m_{1}=\inf _{u \in B_{R}} I(u)<0 .
$$

This completes the proof of Lemma 2.

Theorem 3. Assume $\lambda \in\left(0, \lambda_{0}\right)$, where $\lambda_{0}$ is defined in Lemma 2. Hence, one obtains that problem (1) has a positive solution $u_{0} \in H_{0}^{1}(\Omega)$, with $I\left(u_{0}\right)<0$.

Proof. First, there exists $u_{0} \in B_{R}$, such that $I\left(u_{0}\right)=m_{1}<0$. Actually, from (14), we can obtain that

$$
\begin{gathered}
\frac{1}{2} \int_{\Omega}\left(a|\nabla u|^{2}+|u|^{2}\right) d x+\frac{b}{4}\left(\int_{\Omega}|\nabla u|^{2} d x\right)^{2} \\
-\frac{\mu}{2} \int_{\Omega}|x|^{\alpha-2}|u|^{2} d x-\frac{1}{6} \int_{\Omega} Q(x)\left|u^{+}\right|^{6} d x \geq \rho, \\
\text { for } u \in S_{R}, \\
\frac{1}{2} \int_{\Omega}\left(a|\nabla u|^{2}+|u|^{2}\right) d x+\frac{b}{4}\left(\int_{\Omega}|\nabla u|^{2} d x\right)^{2} \\
-\frac{\mu}{2} \int_{\Omega}|x|^{\alpha-2}|u|^{2} d x-\frac{1}{6} \int_{\Omega} Q(x)\left|u^{+}\right|^{6} d x \geq 0, \\
\text { for } u \in B_{R} .
\end{gathered}
$$

With the definition of $m_{1}$, we have that there exists a minimizing sequence $\left\{u_{n}\right\} \subset B_{R}$ such that $\lim _{n \rightarrow \infty} I\left(u_{n}\right)=$ $m_{1}<0$. Therefore, $\left\{u_{n}\right\}$ is bounded in $B_{R}$. Hence, there exists 
a subsequence, still denoted by $\left\{u_{n}\right\}$. We assume that there exists $u_{0} \in H_{0}^{1}(\Omega)$ such that

$$
\begin{aligned}
u_{n} & \rightarrow u_{0}, \quad \text { weakly in } H_{0}^{1}(\Omega), \\
u_{n} & \longrightarrow u_{0}, \quad \text { strongly in } L^{p}(\Omega)\left(1 \leq p<2^{*}\right), \\
u_{n}(x) & \longrightarrow u_{0}(x), \quad \text { a.e. in } \Omega .
\end{aligned}
$$

For $\gamma \in(0,1)$, by Hölder inequality, one has

$$
\begin{aligned}
& \left|\int_{\Omega} f(x)\left(u_{n}^{+}\right)^{1-\gamma} d x-\int_{\Omega} f(x)\left(u_{0}^{+}\right)^{1-\gamma} d x\right| \\
& \quad \leq \int_{\Omega}|f(x)|\left|\left(u_{n}^{+}\right)^{1-\gamma}-\left(u_{0}^{+}\right)^{1-\gamma}\right| d x \\
& \quad \leq \int_{\Omega}|f(x)|\left|u_{n}^{+}-u_{0}^{+}\right|^{1-\gamma} d x \\
& \quad \leq \int_{\Omega}|f(x)| d x\left|u_{n}^{+}-u_{0}^{+}\right|_{2}^{1-\gamma}|\Omega|^{(1+\gamma) / 2} .
\end{aligned}
$$

Therefore, by (21), we can obtain that

$$
\int_{\Omega} f(x)\left(u_{n}^{+}\right)^{1-\gamma} d x=\int_{\Omega} f(x)\left(u_{0}^{+}\right)^{1-\gamma} d x+o(1) .
$$

Let $\omega_{n}=u_{n}-u_{0}$; by Brézis Lemma, we get

$$
\begin{aligned}
& \int_{\Omega}\left(a\left|\nabla u_{n}\right|^{2}+\left|u_{n}\right|^{2}\right) d x \\
& =\int_{\Omega}\left(a\left|\nabla \omega_{n}\right|^{2}+\left|\omega_{n}\right|^{2}\right) d x \\
& \quad+\int_{\Omega}\left(a\left|\nabla u_{0}\right|^{2}+\left|u_{0}\right|^{2}\right) d x+o(1), \\
& \left(\int_{\Omega}\left|\nabla u_{n}\right|^{2} d x\right)^{2} \\
& =\left(\int_{\Omega}\left|\nabla \omega_{n}\right|^{2} d x\right)^{2}+\left(\int_{\Omega}\left|\nabla u_{0}\right|^{2} d x\right)^{2} \\
& \quad+2 \int_{\Omega}\left|\nabla \omega_{n}\right|^{2} d x \int_{\Omega}\left|\nabla u_{0}\right|^{2} d x+o(1), \\
& \int_{\Omega} Q(x)\left|u_{n}^{+}\right|^{6} d x \\
& =\int_{\Omega} Q(x)\left|\omega_{n}^{+}\right|^{6} d x+\int_{\Omega} Q(x)\left|u_{0}^{+}\right|^{6} d x+o(1), \\
& \int_{\Omega}|x|^{\alpha-2}\left|u_{n}\right|^{2} d x \\
& =\int_{\Omega}|x|^{\alpha-2}\left|\omega_{n}\right|^{2} d x+\int_{\Omega}|x|^{\alpha-2}\left|u_{0}\right|^{2} d x+o(1) .
\end{aligned}
$$

If we set $u_{0}=0$, it follows that $\omega_{n}=u_{n}$ and $\omega_{0} \in B_{R}$. If $u_{0} \neq 0$, by (24), one has $\omega_{n} \in B_{R}$ for $n$ large enough. Therefore, by (20), we have

$$
\begin{array}{r}
\frac{1}{2} \int_{\Omega}\left(a\left|\nabla \omega_{n}\right|^{2}+\left|\omega_{n}\right|^{2}\right) d x+\frac{b}{4}\left(\int_{\Omega}\left|\nabla \omega_{n}\right|^{2} d x\right)^{2} \\
-\frac{\mu}{2} \int_{\Omega}|x|^{\alpha-2}\left|\omega_{n}\right|^{2} d x-\frac{1}{6} \int_{\Omega} Q(x)\left|\omega_{n}^{+}\right|^{6} d x
\end{array}
$$

$\geq 0$.

Hence, noticing (23)-(28), we get

$$
\begin{aligned}
m_{1}= & I\left(u_{n}\right)+o(1) \\
= & I\left(u_{0}\right)+\frac{1}{2} \int_{\Omega}\left(a\left|\nabla \omega_{n}\right|^{2}+\left|\omega_{n}\right|^{2}\right) d x \\
& +\frac{b}{4}\left(\int_{\Omega}\left|\nabla \omega_{n}\right|^{2} d x\right)^{2} \\
& +\frac{b}{2} \int_{\Omega}\left|\nabla \omega_{n}\right|^{2} d x \int_{\Omega}\left|\nabla u_{0}\right|^{2} d x \\
& -\frac{\mu}{2} \int_{\Omega}|x|^{\alpha-2}\left|\omega_{n}\right|^{2} d x-\frac{1}{6} \int_{\Omega} Q(x)\left|\omega_{n}^{+}\right|^{6} d x \\
& +o(1) \\
\geq & I\left(u_{0}\right)+\frac{b}{2} \int_{\Omega}\left|\nabla \omega_{n}\right|^{2} d x \int_{\Omega}\left|\nabla u_{0}\right|^{2} d x+o(1) \\
\geq & I\left(u_{0}\right)+o(1) .
\end{aligned}
$$

Passing to the limit as $n \rightarrow \infty$, we have $m_{1} \geq I\left(u_{0}\right)$. Noticing that $B_{R}$ is closed and convex, so $u_{0} \in B_{R}$. Therefore, from (18), we get $I\left(u_{0}\right)=m_{1}<0$ and $u_{0} \neq 0$. Thus, it can be seen that $u_{0}$ is a local minimizer of $I$.

Next, we prove that $u_{0}$ is a solution of (1) and $u_{0}>0$. By the above expression, we can obtain that $u_{0}$ is a local minimizer of $I$. So, for any $\psi \in H_{0}^{1}(\Omega), \psi \geq 0$, set $t>0$ sufficiently small, such that $u_{0}+t \psi \in B_{R}$; we have

$$
\begin{aligned}
0 \leq & I\left(u_{0}+t \psi\right)-I\left(u_{0}\right)=\frac{1}{2} \\
& \cdot \int_{\Omega}\left(a\left|\nabla\left(u_{0}+t \psi\right)\right|^{2}+\left|u_{0}+t \psi\right|^{2}\right) d x \\
& +\frac{b}{4}\left(\int_{\Omega}\left|\nabla\left(u_{0}+t \psi\right)\right|^{2} d x\right)^{2}-\frac{\mu}{2} \\
& \cdot \int_{\Omega}|x|^{\alpha-2}\left|u_{0}+t \psi\right|^{2} d x-\frac{1}{6} \\
& \cdot \int_{\Omega} Q(x)\left|\left(u_{0}+t \psi\right)^{+}\right|^{6} d x-\frac{\lambda}{1-\gamma} \\
& \cdot \int_{\Omega} f(x)\left|\left(u_{0}+t \psi\right)^{+}\right|^{1-\gamma} d x-\frac{1}{2} \\
& \cdot \int_{\Omega}\left(a\left|\nabla u_{0}\right|^{2}+\left|u_{0}\right|^{2}\right) d x-\frac{b}{4}\left(\int_{\Omega}\left|\nabla u_{0}\right|^{2} d x\right)^{2}
\end{aligned}
$$


Journal of Function Spaces

5

$$
\begin{aligned}
& +\frac{\mu}{2} \int_{\Omega}|x|^{\alpha-2}\left|u_{0}\right|^{2} d x+\frac{1}{6} \int_{\Omega} Q(x)\left|u_{0}^{+}\right|^{6} d x \\
& +\frac{\lambda}{1-\gamma} \int_{\Omega} f(x)\left|u_{0}^{+}\right|^{1-\gamma} d x \leq \frac{1}{2} \\
& \cdot \int_{\Omega}\left(a\left|\nabla\left(u_{0}+t \psi\right)\right|^{2}+\left|u_{0}+t \psi\right|^{2}\right) d x \\
& +\frac{b}{4}\left(\int_{\Omega}\left|\nabla\left(u_{0}+t \psi\right)\right|^{2} d x\right)^{2}-\frac{1}{2} \\
& \cdot \int_{\Omega}\left(a\left|\nabla u_{0}\right|^{2}+\left|u_{0}\right|^{2}\right) d x-\frac{b}{4}\left(\int_{\Omega}\left|\nabla u_{0}\right|^{2} d x\right)^{2} \\
& =\left(\int_{\Omega}\left|\nabla\left(u_{0}+t \psi\right)\right|^{2} d x-\int_{\Omega}\left|\nabla u_{0}\right|^{2} d x\right) \\
& \cdot\left[\frac{a}{2}+\frac{b}{4}\left(\int_{\Omega}\left|\nabla\left(u_{0}+t \psi\right)\right|^{2} d x+\int_{\Omega}\left|\nabla u_{0}\right|^{2} d x\right)\right] \\
& +\frac{1}{2} \int_{\Omega}\left[\left|u_{0}+t \psi\right|^{2}-\left|u_{0}\right|^{2}\right] d x .
\end{aligned}
$$

Currently, we shall prove that $u_{0}$ is a solution of (1). Indeed, by (30), one has

$$
\begin{aligned}
& \frac{\lambda}{1-\gamma} \int_{\Omega} f(x)\left[\left(\left(u_{0}+t \psi\right)^{+}\right)^{1-\gamma}-\left(u_{0}^{+}\right)^{1-\gamma}\right] d x \\
& \leq \frac{a}{2}\left(\int_{\Omega}\left|\nabla\left(u_{0}+t \psi\right)\right|^{2} d x-\int_{\Omega}\left|\nabla u_{0}\right|^{2} d x\right) \\
& +\frac{1}{2}\left(\int_{\Omega}\left|u_{0}+t \psi\right|^{2} d x-\int_{\Omega}\left|u_{0}\right|^{2} d x\right) \\
& +\frac{b}{4}\left[\left(\int_{\Omega}\left|\nabla\left(u_{0}+t \psi\right)\right|^{2} d x\right)^{2}-\left(\int_{\Omega}\left|\nabla u_{0}\right|^{2} d x\right)^{2}\right] \\
& -\frac{\mu}{2}\left[\int_{\Omega}|x|^{\alpha-2}\left|u_{0}+t \psi\right|^{2} d x-\int_{\Omega}|x|^{\alpha-2}\left|u_{0}\right|^{2} d x\right] \\
& -\frac{1}{6} \int_{\Omega} Q(x)\left[\left(\left(u_{0}+t \psi\right)^{+}\right)^{6}-\left(u_{0}^{+}\right)^{6}\right] d x \\
& \quad=\left(\int_{\Omega}\left|\nabla\left(u_{0}+t \psi\right)\right|^{2} d x-\int_{\Omega}\left|\nabla u_{0}\right|^{2} d x\right) \\
& +\int_{\Omega}\left[|x|^{\alpha-2}\left|u_{0}+t \psi\right|^{2}-|x|^{\alpha-2}\left|u_{0}\right|^{2}\right] d x-\frac{1}{6} \\
& \left.+\frac{\int^{2}}{2}+\frac{b}{4}\left(\int_{\Omega}\left|\nabla\left(u_{0}+t \psi\right)\right|^{2} d x+\int_{\Omega}\left|\nabla u_{0}\right|^{2} d x\right)\right]
\end{aligned}
$$

Dividing by $t>0$ and taking the limit $t \rightarrow 0^{+}$, one gets

$$
\begin{aligned}
& \frac{\lambda}{1-\gamma} \liminf _{t \rightarrow 0^{+}} \int_{\Omega} f(x) \frac{\left(\left(u_{0}+t \psi\right)^{+}\right)^{1-\gamma}-\left(u_{0}^{+}\right)^{1-\gamma}}{t} d x \\
& \leq \int_{\Omega}\left(a \nabla u_{0} \nabla \psi+u_{0} \psi\right) d x \\
& \quad+b \int_{\Omega}\left|\nabla u_{0}\right|^{2} d x \int_{\Omega} \nabla u_{0} \nabla \psi d x \\
& \quad-\mu \int_{\Omega}|x|^{\alpha-2} u_{0} \psi d x-\int_{\Omega} Q(x)\left(u_{0}^{+}\right)^{5} \psi d x .
\end{aligned}
$$

Note that

$$
\begin{aligned}
& \frac{\lambda}{1-\gamma} \int_{\Omega} f(x) \frac{\left(\left(u_{0}+t \psi\right)^{+}\right)^{1-\gamma}-\left(u_{0}^{+}\right)^{1-\gamma}}{t} d x \\
& =\lambda \int_{\Omega} f(x)\left(\left(u_{0}^{+}+\xi t \psi\right)^{+}\right)^{-\gamma} \psi d x,
\end{aligned}
$$

where $\xi \rightarrow 0^{+}$and $\left(\left(u_{0}+\xi t \psi\right)^{+}\right)^{-\gamma} \psi \rightarrow\left(u_{0}^{+}\right)^{-\gamma} \psi$ ae. $x \in \Omega$ as $t \rightarrow 0^{+}$, for $\left(\left(u_{0}+\xi t \psi\right)^{+}\right)^{-\gamma} \psi \geq 0$. Therefore, using Fatou Lemma, one has

$$
\begin{aligned}
& \lambda \int_{\Omega} f(x)\left(u_{0}^{+}\right)^{-\gamma} \psi d x \leq \frac{\lambda}{1-\gamma} \\
& \quad \cdot \liminf _{t \rightarrow 0^{+}} \int_{\Omega} f(x) \frac{\left(\left(u_{0}+t \psi\right)^{+}\right)^{1-\gamma}-\left(u_{0}^{+}\right)^{1-\gamma}}{t} d x .
\end{aligned}
$$

Hence, by (32) and the above estimate, we can obtain that

$$
\begin{aligned}
& \int_{\Omega}\left(a \nabla u_{0} \nabla \psi+u_{0} \psi\right) d x \\
& \quad+b \int_{\Omega}\left|\nabla u_{0}\right|^{2} d x \int_{\Omega} \nabla u_{0} \nabla \psi d x \\
& \quad-\mu \int_{\Omega}|x|^{\alpha-2} u_{0} \psi d x-\int_{\Omega} Q(x)\left(u_{0}^{+}\right)^{5} \psi d x \\
& \quad-\lambda \int_{\Omega} f(x)\left(u_{0}\right)^{-\gamma} \psi d x \geq 0, \quad \psi \geq 0 .
\end{aligned}
$$

For $I\left(u_{0}\right)<0$ and (14), we can get $u_{0} \notin S_{R}$; then, $\int_{\Omega}\left|\nabla u_{0}\right| d x<$ $R$. For $u_{0}$, there is $\delta_{1} \in(0,1)$ such that $(1+t) u_{0} \in B_{R}$ for $|t| \leq \delta_{1}$. Define $k:\left[-\delta_{1}, \delta_{1}\right] \rightarrow \mathbb{R}$ by $k(t)=I\left((1+t) u_{0}\right)$. Actually, when $t=0, k(t)$ achieves its minimum; namely,

$$
\begin{aligned}
\left.k^{\prime}(t)\right|_{t=0}= & \int_{\Omega}\left(a\left|\nabla u_{0}\right|^{2}+\left|u_{0}\right|^{2}\right) d x \\
& +b\left(\int_{\Omega}\left|\nabla u_{0}\right|^{2} d x\right)^{2} \\
& -\mu \int_{\Omega}|x|^{\alpha-2}\left|u_{0}\right|^{2} d x \\
& -\int_{\Omega} Q(x)\left|u_{0}^{+}\right|^{6} d x \\
& -\lambda \int_{\Omega} f(x)\left|u_{0}^{+}\right|^{1-\gamma} d x=0 .
\end{aligned}
$$


Assume $\phi \in H_{0}^{1}(\Omega)$ and $\varepsilon>0$. Define $\Psi \in H_{0}^{1}(\Omega)$ by $\Psi=$ $\left(u_{0}^{+}+\varepsilon \phi\right)^{+}$. So, from (35) and (36), we can get

$$
\begin{aligned}
& 0 \leq \int_{\Omega}\left[a \nabla u_{0} \nabla \Psi+u_{0} \Psi+b\left|\nabla u_{0}\right|^{2} \nabla u_{0} \nabla \Psi\right. \\
& -\mu|x|^{\alpha-2} u_{0} \Psi-Q(x)\left(u^{+}\right)^{5} \Psi \\
& \left.-\lambda f(x)\left(u_{0}^{+}\right)^{-\gamma} \Psi\right] d x \\
& =\int_{\left\{u_{0}^{+}+\varepsilon \phi>0\right\}}\left[a \nabla u_{0} \nabla\left(u_{0}^{+}+\varepsilon \phi\right)+u_{0}\left(u_{0}^{+}+\varepsilon \phi\right)\right. \\
& \left.+b\left|\nabla u_{0}\right|^{2} \nabla u_{0} \nabla\left(u_{0}^{+}+\varepsilon \phi\right)\right] d x \\
& -\int_{\left\{u_{0}^{+}+\varepsilon \phi>0\right\}}\left[\mu|x|^{\alpha-2} u_{0}\left(u_{0}^{+}+\varepsilon \phi\right)\right. \\
& +Q(x)\left(u_{0}^{+}\right)^{5}\left(u_{0}^{+}+\varepsilon \phi\right) \\
& \left.+\lambda f(x)\left(u_{0}^{+}\right)^{-\gamma}\left(u_{0}^{+}+\varepsilon \phi\right)\right] d x=\left(\int_{\Omega}\right. \\
& \left.-\int_{\left\{u_{0}^{+}+\varepsilon \phi \leq 0\right\}}\right)\left[a \nabla u_{0} \nabla\left(u_{0}^{+}+\varepsilon \phi\right)+u_{0}\left(u_{0}^{+}+\varepsilon \phi\right)\right. \\
& +b\left|\nabla u_{0}\right|^{2} \nabla u_{0} \nabla\left(u_{0}^{+}+\varepsilon \phi\right)-\mu|x|^{\alpha-2} u_{0}\left(u_{0}^{+}+\varepsilon \phi\right) \\
& -Q(x)\left(u_{0}^{+}\right)^{5}\left(u_{0}^{+}+\varepsilon \phi\right)-\lambda f(x)\left(u_{0}^{+}\right)^{-\gamma}\left(u_{0}^{+}\right. \\
& +\varepsilon \phi)] d x \leq \int_{\Omega}\left(a\left|\nabla u_{0}\right|^{2}+\left|u_{0}\right|^{2}\right) d x \\
& +b\left(\int_{\Omega}\left|\nabla u_{0}\right|^{2} d x\right)^{2}-\mu \int_{\Omega}|x|^{\alpha-2}\left|u_{0}\right|^{2} d x \\
& -\int_{\Omega} Q(x)\left|u_{0}^{+}\right|^{6} d x-\lambda \int_{\Omega} f(x)\left|u_{0}^{+}\right|^{1-\gamma} d x \\
& +\varepsilon \int_{\Omega}\left[a \nabla u_{0} \nabla \phi+u_{0} \phi+b\left|\nabla u_{0}\right|^{2} \nabla u_{0} \nabla \phi\right. \\
& -\mu|x|^{\alpha-2} u_{0} \phi-Q(x)\left(u_{0}^{+}\right)^{5} \phi \\
& \left.-\lambda f(x)\left(u_{0}^{+}\right)^{-\gamma} \phi\right] d x \\
& -\int_{\left\{u_{0}^{+}+\varepsilon \phi \leq 0\right\}}\left[a \nabla u_{0} \nabla\left(u_{0}^{+}+\varepsilon \phi\right)+u_{0}\left(u_{0}^{+}+\varepsilon \phi\right)\right. \\
& \left.+b\left|\nabla u_{0}\right|^{2} \nabla u_{0} \nabla\left(u_{0}^{+}+\varepsilon \phi\right)\right] d x \\
& +\int_{\left\{u_{0}^{+}+\varepsilon \phi \leq 0\right\}}\left[\mu|x|^{\alpha-2} u_{0}\left(u_{0}^{+}+\varepsilon \phi\right)\right. \\
& +Q(x)\left(u_{0}^{+}\right)^{5}\left(u_{0}^{+}+\varepsilon \phi\right) \\
& \left.+\lambda f(x)\left(u_{0}^{+}\right)^{-\gamma}\left(u_{0}^{+}+\varepsilon \phi\right)\right] d x \\
& \leq \varepsilon \int_{\Omega}\left[a \nabla u_{0} \nabla \phi+u_{0} \phi+b\left|\nabla u_{0}\right|^{2} \nabla u_{0} \nabla \phi\right.
\end{aligned}
$$

$$
\begin{aligned}
& -\mu|x|^{\alpha-2} u_{0} \phi-Q(x)\left(u_{0}^{+}\right)^{5} \phi \\
& \left.-\lambda f(x)\left(u_{0}^{+}\right)^{-\gamma} \phi\right] d x-\varepsilon \int_{\left\{u_{0}^{+}+\varepsilon \phi \leq 0\right\}}[a \\
& \left.+b\left|\nabla u_{0}\right|^{2} \nabla u_{0} \nabla \phi+u_{0} \phi\right] d x .
\end{aligned}
$$

By the measure of the domain of integration $\left\{u_{0}^{+}+\varepsilon \phi \leq 0\right\} \rightarrow$ 0 as $\varepsilon \rightarrow 0$, it can be seen that

$$
\lim _{\varepsilon \rightarrow 0} \int_{\left\{u_{0}^{+}+\varepsilon \phi \leq 0\right\}}\left[a+b\left|\nabla u_{0}\right|^{2} \nabla u_{0} \nabla \phi+u_{0} \phi\right] d x=0 .
$$

Then, dividing by $\varepsilon$ and letting $\varepsilon \rightarrow 0$ in (37), one has

$$
\begin{aligned}
(a+ & \left.b \int_{\Omega}\left|\nabla u_{0}\right|^{2} d x\right) \int_{\Omega} \nabla u_{0} \nabla \phi d x+\int_{\Omega} u_{0} \phi d x \\
& -\mu \int_{\Omega}|x|^{\alpha-2} u_{0} \phi d x-\int_{\Omega} Q(x)\left|u_{0}^{+}\right|^{5} \phi d x \\
& -\lambda \int_{\Omega} f(x)\left|u_{0}^{+}\right|^{-\gamma} \phi d x \geq 0 .
\end{aligned}
$$

With the arbitrariness of $\phi$, this inequality also holds for $-\phi$; that is,

$$
\begin{aligned}
(a+ & \left.b \int_{\Omega}\left|\nabla u_{0}\right|^{2} d x\right) \int_{\Omega} \nabla u_{0} \nabla \phi d x+\int_{\Omega} u_{0} \phi d x \\
& -\mu \int_{\Omega}|x|^{\alpha-2} u_{0} \phi d x-\int_{\Omega} Q(x)\left|u_{0}^{+}\right|^{5} \phi d x \\
& -\lambda \int_{\Omega} f(x)\left|u_{0}^{+}\right|^{-\gamma} \phi d x=0 .
\end{aligned}
$$

On the one hand, we can take the test function $\phi=u_{0}^{-}$ in (40); we have $\int_{\Omega}\left|\nabla u_{0}^{-}\right| d x=0$, and then we get $u_{0} \geq 0$. Therefore, $u_{0}$ is a nonzero solution of (1).

On the other hand, let $\left\{u_{\alpha}\right\}$ be a family of positive solutions of (12); with an easy computation, we see that

$$
\begin{aligned}
& -\left(a+b \int_{\Omega}\left|\nabla u_{\alpha}\right|^{2} d x\right) \Delta u_{\alpha}+u_{\alpha} \\
& =Q(x) u_{\alpha}^{5}+\mu|x|^{\alpha-2} u_{\alpha}+\frac{\lambda f(x)}{\left(u_{\alpha}+\alpha\right)^{\gamma}} \\
& \geq \min \left\{1, Q_{m}\right\} \min \left\{1, \frac{\lambda f_{m}}{2^{\gamma}}\right\},
\end{aligned}
$$

where $Q_{m}=\min Q(x), f_{m}=\min f(x)$, which means that $u_{0} \in H_{0}^{1}(\Omega)$ and satisfies

$$
-\Delta u_{0} \geq \frac{\min \left\{1, Q_{m}\right\} \min \left\{1, \lambda f_{m} / 2\right\}-u_{0}}{a+b \int_{\Omega}\left|\nabla u_{0}\right|^{2} d x}, \quad \text { in } \Omega .
$$

Noticing $u_{0} \geq 0, u_{0} \neq 0$, thus, we have $u_{0}>0$, in $\Omega$ by the strong maximum principle.

From the above discussion, we can deduce that $u_{0}$ is a positive solution of $(1)$ with $I\left(u_{0}\right)=m_{1}<0$. This completes the proof of Theorem 3 . 


\section{Existence of a Mountain-Pass Solution of Problem (12)}

In this section, we consider the existence of at least one mountain-pass solution of the perturbation problem.

Lemma 4. Suppose that $\lambda<\lambda_{0}$. For $R, \rho>0$, where $\lambda_{0}$, $R$, and $\rho$ are as in Lemma 2, the functional $I_{\alpha}$ satisfies the following conditions:

(i) $I_{\alpha}(u) \geq \rho>0$, if $u \in S_{R}$.

(ii) There exists $u_{1} \in H_{0}^{1}(\Omega)$ such that $\int_{\Omega}\left|\nabla u_{1}\right| d x>R$, and $I_{\alpha}\left(u_{1}\right)<\rho$.

Proof. (i) Notice the subadditivity of $t^{1-\gamma}$; namely,

$$
\left(u^{+}+\alpha\right)^{1-\gamma}-\alpha^{1-\gamma} \leq\left(u^{+}\right)^{1-\gamma}, \quad \forall u \in H_{0}^{1}(\Omega) .
$$

From (43), we can get $I_{\alpha}(u) \geq I(u), \forall u \in H_{0}^{1}(\Omega)$. Hence, when $\lambda<\lambda_{0}$, from (14), we can get the conclusion. has

(ii) For every $u^{+} \in H_{0}^{1}(\Omega), u^{+} \neq 0$, and $t>0$, one

$$
\begin{aligned}
& I_{\alpha}(t u) \\
& =\frac{t^{2}}{2} \int_{\Omega}\left(a|\nabla u|^{2}+|u|^{2}\right) d x+\frac{b}{4} t^{4}\left(\int_{\Omega}|\nabla u|^{2} d x\right)^{2} \\
& \quad-\frac{\mu}{2} \int_{\Omega}|x|^{\alpha-2}|u|^{2} d x-\frac{t^{6}}{6} \int_{\Omega} Q(x)\left(u^{+}\right)^{6} d x \\
& \quad-\frac{\lambda}{1-\gamma} \int_{\Omega} f(x)\left[\left(t u^{+}+\alpha\right)^{1-\gamma}-\alpha^{1-\gamma}\right] d x \\
& \leq \frac{t^{2}}{2} \int_{\Omega}\left(a|\nabla u|^{2}+|u|^{2}\right) d x+\frac{b}{4} t^{4}\left(\int_{\Omega}|\nabla u|^{2} d x\right)^{2} \\
& \quad-\frac{t^{6}}{6} \int_{\Omega} Q(x)\left(u^{+}\right)^{6} d x \longrightarrow-\infty,
\end{aligned}
$$

as $t \rightarrow+\infty$. Hence, we can consider $u_{1}$ such that $\int_{\Omega}\left|\nabla u_{1}\right| d x>R$ and $I_{\alpha}\left(u_{1}\right)<\rho$. This completes the proof of Lemma 4.

We define

$$
\begin{aligned}
\Lambda= & \frac{a b S^{3}}{6 Q_{M}}+\frac{b^{3} S^{6}}{24 Q_{M}^{2}}+\frac{a S \sqrt{b^{2} S^{4}+4 a S Q_{M}}}{6 Q_{M}} \\
& +\frac{b^{2} S^{4} \sqrt{b^{2} S^{4}+4 a S Q_{M}}}{24 Q_{M}^{2}} .
\end{aligned}
$$

Lemma 5. Let $\alpha \in(0,1)$; then $I_{\alpha}$ satisfies the $(P S)_{c}$ condition for every $c<\Lambda-D \lambda^{2 /(1+\gamma)}$, where $D=$ $(1+\gamma)\left(3^{(1-\gamma) / 2}|\Omega|^{(5+\gamma) / 6}\left(\mu_{1}(1-\gamma)\right)^{(1-\gamma) / 2} f_{m} / 2^{(2-\gamma) / 2}\left(\left(a \mu_{1}-\right.\right.\right.$ $\left.\mu) S)^{(1-\gamma) / 2}\right)^{2 /(1+\gamma)}$, and $S$ is as in (9).
Proof. Let $\left\{v_{n}\right\} \subset H_{0}^{1}(\Omega)$, so

$$
\begin{aligned}
& I_{\alpha}\left(v_{n}\right) \longrightarrow c, \\
& I_{\alpha}^{\prime}\left(v_{n}\right) \longrightarrow 0,
\end{aligned}
$$

as $n \longrightarrow \infty$.

We shall claim that $\left\{v_{n}\right\}$ is bounded in $H_{0}^{1}(\Omega)$. Otherwise, we can suppose that $\int_{\Omega}\left|\nabla v_{n}\right| d x \rightarrow \infty$, as $n \rightarrow \infty$. From (46), (43), and (15), one has

$$
\begin{aligned}
1+ & +o(1) \int_{\Omega}\left|\nabla v_{n}\right| d x \geq I_{\alpha}\left(v_{n}\right)-\frac{1}{6}\left\langle I_{\alpha}^{\prime}\left(v_{n}\right), v_{n}\right\rangle \\
= & \frac{1}{3} \int_{\Omega}\left(a\left|\nabla v_{n}\right|^{2}+\left|v_{n}\right|^{2}\right) d x \\
& +\frac{b}{12}\left(\int_{\Omega}\left|\nabla v_{n}\right|^{2} d x\right)^{2}-\frac{\mu}{3} \int_{\Omega}|x|^{\alpha-2}\left|v_{n}\right|^{2} d x \\
& -\frac{\lambda}{1-\gamma} \int_{\Omega} f(x)\left[\left(v_{n}^{+}+\alpha\right)^{1-\gamma}-\alpha^{1-\gamma}\right] d x \\
& +\frac{\lambda}{6} \int_{\Omega} f(x) \frac{v_{n}}{\left(v_{n}^{+}+\alpha\right)^{\gamma}} d x \\
\geq & \frac{a}{3} \int_{\Omega}\left|\nabla v_{n}\right|^{2} d x+\frac{b}{12}\left(\int_{\Omega}\left|\nabla v_{n}\right|^{2} d x\right)^{2} \\
& -\frac{\mu}{3} \int_{\Omega}|x|^{\alpha-2}\left|v_{n}\right|^{2} d x \\
& -\frac{\lambda}{1-\gamma}|\Omega|^{(5+\gamma) / 6} S^{-(1-\gamma) / 2} \int_{\Omega} f(x)\left|\nabla v_{n}^{+}\right|^{1-\gamma} d x \\
& \frac{1}{3}\left(a-\gamma(x)\left(v_{n}^{+}\right)^{1-\gamma} d x\right. \\
\mu_{1} & \mu \int_{\Omega}\left|\nabla v_{n}\right|^{2} d x+\frac{b}{12}\left(\int_{\Omega}\left|\nabla v_{n}\right|^{2} d x\right)^{2} \\
& \frac{\lambda}{2} d x
\end{aligned}
$$

By $1-\gamma<2<4$, it can be seen that the last inequality is unreasonable. Clearly, $\left\{v_{n}\right\}$ is bounded in $H_{0}^{1}(\Omega)$. Up to a subsequence, there exists $v_{*} \in H_{0}^{1}(\Omega)$ such that

$$
\begin{gathered}
v_{n} \rightarrow v_{*}, \quad \text { weakly in } H_{0}^{1}(\Omega), \\
v_{n} \longrightarrow v_{*}, \\
\text { strongly in } L^{p}(\Omega)(1 \leq p<6), \\
v_{n}(x) \longrightarrow v_{*}(x), \quad \text { a.e. in } \Omega,
\end{gathered}
$$

there exists $k \in L^{1}(\Omega)$ such that for all $n$,

$$
\left|v_{n}(x)\right| \leq k(x) \text { a.e. in } \Omega \text {. }
$$


Since $\alpha>0$ and $\left|v_{*}\right| /\left(v_{*}+\alpha\right)^{\gamma} \leq\left|v_{*}(x)\right| / \alpha^{\gamma}$, using the dominated convergence theorem and (48), we get

$$
\begin{array}{r}
\lim _{n \rightarrow \infty} \int_{\Omega} f(x)\left(v_{n}^{+}+\alpha\right)^{-\gamma} v_{*} d x \\
=\int_{\Omega} f(x)\left(v_{*}^{+}+\alpha\right)^{-\gamma} v_{*} d x .
\end{array}
$$

Furthermore, from $\alpha>0$ and (48), we get

$$
\left|\frac{v_{n}}{\left(v_{n}^{+}+\alpha\right)^{\gamma}}\right| \leq \frac{\left|v_{n}\right|}{\left(v_{n}^{+}+\alpha\right)^{\gamma}} \leq \frac{\left|v_{n}\right|}{\alpha^{\gamma}} \leq \frac{1}{\alpha^{\gamma}} k(x) .
$$

Hence, it follows from the dominated convergence theorem that

$$
\begin{array}{r}
\lim _{n \rightarrow \infty} \int_{\Omega} f(x)\left(v_{n}^{+}+\alpha\right)^{-\gamma} v_{n} d x \\
=\int_{\Omega} f(x)\left(v_{*}^{+}+\alpha\right)^{-\gamma} v_{*} d x .
\end{array}
$$

Let $\omega_{n}=v_{n}-v_{*}$; then, $\int_{\Omega}\left|\nabla \omega_{n}\right| d x \rightarrow 0$ as $n \rightarrow \infty$. Otherwise, there exists a subsequence (still denoted by $\omega_{n}$ ) such that $\lim _{n \rightarrow \infty} \int_{\Omega}\left|\nabla \omega_{n}\right| d x=l$ (where $l$ is a positive constant). Therefore, by $\lim _{n \rightarrow \infty}\left\langle I_{\alpha}^{\prime}\left(v_{n}\right), v_{*}\right\rangle=0$ and (49), one has

$$
\begin{aligned}
& \int_{\Omega}\left(a\left|\nabla v_{*}\right|^{2}+\left|v_{*}\right|^{2}\right) d x+b\left(\int_{\Omega}\left|\nabla v_{*}\right|^{2} d x\right)^{2} \\
& \quad+b l^{2} \int_{\Omega}\left|\nabla v_{*}\right|^{2} d x-\mu \int_{\Omega}|x|^{\alpha-2}\left|v_{*}\right|^{2} d x \\
& \quad-\int_{\Omega} Q(x)\left(v_{*}^{+}\right)^{6} d x \\
& -\lambda \int_{\Omega} f(x)\left(v_{*}^{+}+\alpha\right)^{-\gamma} v_{*} d x=0 .
\end{aligned}
$$

By Brézis-Lieb Lemma and (46), we get

$$
\begin{gathered}
\int_{\Omega}\left(a\left|\nabla v_{*}\right|^{2}+\left|v_{*}\right|^{2}\right) d x+\int_{\Omega}\left(a\left|\nabla \omega_{n}\right|^{2}+\left|\omega_{n}\right|^{2}\right) d x \\
+b\left(\int_{\Omega}\left|\nabla v_{*}\right|^{2} d x\right)^{2}+b\left(\int_{\Omega}\left|\nabla \omega_{n}\right|^{2} d x\right)^{2} \\
+2 b \int_{\Omega}\left|\nabla \omega_{n}\right|^{2} d x \int_{\Omega}\left|\nabla v_{*}\right|^{2} d x \\
-\mu \int_{\Omega}|x|^{\alpha-2}\left|v_{*}\right|^{2} d x-\mu \int_{\Omega}|x|^{\alpha-2}\left|\omega_{n}\right|^{2} d x \\
-\int_{\Omega} Q(x)\left(v_{*}^{+}\right)^{6} d x-\int_{\Omega} Q(x)\left(\omega_{n}^{+}\right)^{6} d x \\
-\lambda \int_{\Omega} f(x)\left(v_{n}^{+}+\alpha\right)^{-\gamma} v_{n} d x=o(1) .
\end{gathered}
$$

Thus, from the above equality and (51) and (52), we get

$$
\begin{aligned}
\int_{\Omega} & \left(a\left|\nabla \omega_{n}\right|^{2}+\left|\omega_{n}\right|^{2}\right) d x+b\left(\int_{\Omega}\left|\nabla \omega_{n}\right|^{2} d x\right)^{2} \\
& +b \int_{\Omega}\left|\nabla \omega_{n}\right|^{2} d x \int_{\Omega}\left|\nabla v_{*}\right|^{2} d x \\
& -\mu \int_{\Omega}|x|^{\alpha-2}\left|\omega_{n}\right|^{2} d x-\int_{\Omega} Q(x)\left(\omega_{n}^{+}\right)^{6} d x \\
= & o(1) .
\end{aligned}
$$

For $\int_{\Omega}\left(\omega_{n}^{+}\right)^{6} d x \leq \int_{\Omega}\left|\omega_{n}\right|^{6} d x$, by (54) and (9), one has

$$
\begin{aligned}
\int_{\Omega} & {\left[\left(a-\frac{\mu}{\mu_{1}}\right)\left|\nabla \omega_{n}\right|^{2}+\left|\omega_{n}\right|^{2}\right] d x } \\
& +b\left(\int_{\Omega}\left|\nabla \omega_{n}\right|^{2} d x\right)^{2} \\
& +b \int_{\Omega}\left|\nabla \omega_{n}\right|^{2} d x \int_{\Omega}\left|\nabla v_{*}\right|^{2} d x \\
\leq & S^{-3} \int_{\Omega} Q(x)\left|\nabla \omega_{n}\right|^{6} d x+o(1) .
\end{aligned}
$$

Taking the limit $n \rightarrow \infty$, we have

$$
\begin{aligned}
& \left(a-\frac{\mu}{\mu_{1}}\right) l^{2}+b l^{4}+b l^{2} \int_{\Omega}\left|\nabla v_{*}\right|^{2} d x \\
& \leq S^{-3} l^{6} \int_{\Omega} Q(x) d x,
\end{aligned}
$$

and then we get

$$
\geq \frac{b S^{3}+\sqrt{b^{2} S^{6}+4 S^{3} \int_{\Omega} Q(x) d x\left(a-\mu / \mu_{1}+b \int_{\Omega}\left|\nabla v_{*}\right|^{2} d x\right)}}{2 \int_{\Omega} Q(x) d x} .
$$

Furthermore, from Brézis-Lieb Lemma and (46) and (54) and letting $n \rightarrow \infty$, we obtain that

$$
\begin{aligned}
I_{\alpha}\left(v_{*}\right)= & c-\frac{1}{3}\left(a-\frac{\mu}{\mu_{1}}\right) l^{2}-\frac{b}{12} l^{4} \\
& -\frac{b}{3} l^{2} \int_{\Omega}\left|\nabla v_{*}\right|^{2} d x-\frac{1}{3} \int_{\Omega}\left|\omega_{n}\right|^{2} d x .
\end{aligned}
$$

On the one hand, using (54), (43), and (15) and Young's inequality, one has

$$
\begin{aligned}
& I_{\alpha}\left(v_{*}\right)=\frac{1}{2} \int_{\Omega}\left(a\left|\nabla v_{*}\right|^{2}+\left|v_{*}\right|^{2}\right) d x \\
& \quad+\frac{b}{4}\left(\int_{\Omega}\left|\nabla v_{*}\right|^{2} d x\right)^{2}-\frac{\mu}{2} \int_{\Omega}|x|^{\alpha-2}\left|v_{*}\right|^{2} d x-\frac{1}{6}
\end{aligned}
$$




$$
\begin{aligned}
& \int_{\Omega} Q(x)\left|v_{*}^{+}\right|^{6} d x-\frac{\lambda}{1-\gamma} \int_{\Omega} f(x) \\
& \cdot\left(\left(v_{*}^{+}+\alpha\right)^{1-\gamma}-\alpha^{1-\gamma}\right) d x \geq \frac{1}{3} \\
& \cdot \int_{\Omega}\left(a\left|\nabla v_{*}\right|^{2}+\left|v_{*}\right|^{2}\right) d x+\frac{b}{12}\left(\int_{\Omega}\left|\nabla v_{*}\right|^{2} d x\right)^{2} \\
& -\frac{b}{6} l^{2} \int_{\Omega}\left|\nabla v_{*}\right|^{2} d x-\frac{\mu}{3} \int_{\Omega}|x|^{\alpha-2}\left|v_{*}\right|^{2} d x-\frac{\lambda}{1-\gamma} \\
& \cdot \int_{\Omega} f(x)\left(\left(v_{*}^{+}+\alpha\right)^{1-\gamma}-\alpha^{1-\gamma}\right) d x \geq \frac{a}{3} \\
& \cdot \int_{\Omega}\left|\nabla v_{*}\right|^{2} d x-\frac{\mu}{3} \int_{\Omega}|x|^{\alpha-2}\left|v_{*}\right|^{2} d x-\frac{\lambda}{1-\gamma} \\
& \cdot \int_{\Omega} f(x)\left(v_{*}\right)^{1-\gamma} d x-\frac{b}{4} l^{2} \int_{\Omega}\left|\nabla v_{*}\right|^{2} d x \geq \frac{1}{3}(a \\
& \left.-\frac{\mu}{\mu_{1}}\right) \int_{\Omega}\left|\nabla v_{*}\right|^{2} d x-\frac{\lambda}{1-\gamma}|\Omega|^{(5+\gamma) / 6} \\
& \cdot S^{-(1-\gamma) / 2} \int_{\Omega} f(x)\left|\nabla v_{*}\right|^{1-\gamma} d x-\frac{b}{4} l^{2} \int_{\Omega}\left|\nabla v_{*}\right|^{2} d x
\end{aligned}
$$

$$
\begin{aligned}
& \geq \frac{\mu(a-1)}{3 \mu_{1}} \int_{\Omega}\left|\nabla v_{*}\right|^{2} d x \\
& -\frac{1-\gamma}{2}\left[\left(\frac{2\left(a \mu_{1}-\mu\right)}{3 \mu_{1}(1-\gamma)}\right)^{(1-\gamma) / 2}\right. \\
& \left.\cdot \int_{\Omega}\left|\nabla v_{*}\right|^{1-\gamma} d x\right]^{2 /(1-\gamma)} \\
& -\frac{1+\gamma}{2}\left[\frac{\lambda|\Omega|^{(5+\gamma) / 6}\left(3 \mu_{1}(1-\gamma)\right)^{(1-\gamma) / 2} f_{m}}{\left(2\left(a \mu_{1}-\mu\right) S\right)^{(1-\gamma) / 2}}\right]^{2 /(1+\gamma)} \\
& -\frac{b}{4} l^{2} \int_{\Omega}\left|\nabla v_{*}\right|^{2} d x=-D \lambda^{2 /(1+\gamma)}-\frac{b}{4} \\
& \cdot l^{2} \int_{\Omega}\left|\nabla v_{*}\right|^{2} d x .
\end{aligned}
$$

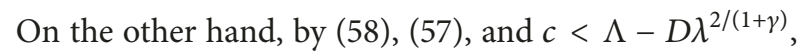
one has

$$
\begin{aligned}
& I_{\alpha}\left(v_{*}\right) \leq c-\frac{1}{3}\left(a-\frac{\mu}{\mu_{1}}\right) l^{2}-\frac{1}{12} b l^{4}-\frac{1}{3} b l^{2} \int_{\Omega}\left|\nabla v_{*}\right|^{2} d x \\
& \leq c-\frac{1}{3}\left(a-\frac{\mu}{\mu_{1}}\right) \frac{b S^{3}+\sqrt{b^{2} S^{6}+4 S^{3} Q_{M}\left(a-\mu / \mu_{1}+b \int_{\Omega}\left|\nabla v_{*}\right|^{2} d x\right)}}{2 Q_{M}} \\
& -\frac{b}{12} \frac{b^{2} S^{6}+2 S^{3} Q_{M}\left(a-\mu / \mu_{1}+b \int_{\Omega}\left|\nabla v_{*}\right|^{2} d x\right)+b S^{4} \sqrt{b^{2} S^{4}+4 S Q_{M}\left(a-\mu / \mu_{1}+b \int_{\Omega}\left|\nabla v_{*}\right|^{2} d x\right)}}{2\left(Q_{M}\right)^{2}} \\
& -\frac{b}{3} \int_{\Omega}\left|\nabla v_{*}\right|^{2} d x \frac{b S^{3}+\sqrt{b^{2} S^{6}+4 S^{3} Q_{M}\left(a-\mu / \mu_{1}+b \int_{\Omega}\left|\nabla v_{*}\right|^{2} d x\right)}}{2 Q_{M}} \\
& \leq c-\frac{a b S^{3}}{6 Q_{M}}-\frac{b^{3} S^{6}}{24 Q_{M}^{2}}-\frac{a S \sqrt{b^{2} S^{4}+4 S^{3} Q_{M}\left(a-\mu / \mu_{1}+b \int_{\Omega}\left|\nabla v_{*}\right|^{2} d x\right)}}{6 Q_{M}} \\
& -\frac{b^{2} S^{4} \sqrt{b^{2} S^{4}+4 S^{3} Q_{M}\left(a-\mu / \mu_{1}+b \int_{\Omega}\left|\nabla v_{*}\right|^{2} d x\right)}}{24 Q_{M}^{2}} \\
& \leq c-\frac{a b S^{3}}{6 Q_{M}}-\frac{b^{3} S^{6}}{24 Q_{M}^{2}}-\frac{a S \sqrt{b^{2} S^{4}+4 a S Q_{M}}}{6 Q_{M}}-\frac{b^{2} S^{4} \sqrt{b^{2} S^{4}+4 a S Q_{M}}}{24 Q_{M}^{2}}-\frac{b}{4} l^{2} \int_{\Omega}\left|\nabla v_{*}\right|^{2} d x \\
& =c-\Lambda-\frac{b}{4} l^{2} \int_{\Omega}\left|\nabla v_{*}\right|^{2} d x<-D \lambda^{2 /(1+\gamma)}-\frac{b}{4} l^{2} \int_{\Omega}\left|\nabla v_{*}\right|^{2} d x,
\end{aligned}
$$

which contradicts inequality (59). Then, it follows that $v_{n} \rightarrow$ $v_{*}$ in $H_{0}^{1}(\Omega)$ as $n \rightarrow \infty$. From the above discussion, $I_{\alpha}$ satisfies the $(P S)_{c}$ condition. This completes the proof of Lemma 5.
For all $\varepsilon>0$, we consider $u_{\varepsilon}(x)=(3 \varepsilon)^{1 / 4} /\left(\varepsilon+|x|^{2}\right)^{1 / 2}$, $x \in \mathbb{R}^{N}$, which is a solution of the critical problem

$$
-\Delta u_{\varepsilon}=u_{\varepsilon}^{5}, \quad \text { in } \mathbb{R}^{N} .
$$


Let

$$
\begin{aligned}
C_{\varepsilon} & =(3 \varepsilon)^{1 / 4}, \\
y_{\varepsilon}(x) & =\frac{u_{\varepsilon}(x)}{C_{\varepsilon}} .
\end{aligned}
$$

Pick a cut-off function $\varphi \in C_{0}^{\infty}(\Omega)$ such that $\varphi(x)=1$ for $|x|<R_{0}$, and $\varphi(x)=0$ for $|x|>2 R_{0}, 0 \leq \varphi(x) \leq 1$. Set $\omega_{\varepsilon}(x)=\varphi(x) y_{\varepsilon}(x), v_{\varepsilon}(x)=\omega_{\varepsilon}(x) /\left|\omega_{\varepsilon}\right|^{6} ;$ then, $\left|v_{\varepsilon}\right|_{6}^{6}=1$. Thus, we have the following results (see the details in [25]):

$$
\begin{aligned}
\int_{\Omega}\left|\nabla v_{\varepsilon}\right|^{2} d x & \leq S+C \varepsilon^{1 / 2}, \\
\left(\int_{\Omega}\left|\nabla v_{\varepsilon}\right|^{2} d x\right)^{3} & \leq S^{3}+C \varepsilon^{1 / 2}, \\
\left(\int_{\Omega}\left|\nabla v_{\varepsilon}\right|^{2} d x\right)^{4} & \leq S^{4}+C \varepsilon^{1 / 2}, \\
\left(\int_{\Omega}\left|\nabla v_{\varepsilon}\right|^{2} d x\right)^{6} & \leq S^{6}+C \varepsilon^{1 / 2}, \\
O\left(\varepsilon^{(1-\gamma) / 4}\right) & \leq \int_{\Omega}\left|v_{\varepsilon}\right|^{1-\gamma} d x \leq O\left(\varepsilon^{(1-\gamma) / 4}\right), \\
\int_{\Omega}|x|^{\alpha-2}\left|v_{\varepsilon}\right|^{1-\gamma} d x & =O\left(\varepsilon^{\alpha / 2}\right) .
\end{aligned}
$$

Lemma 6. Since $\alpha \in(0,1)$ and $\lambda>0$ sufficiently small, there exists $u_{1} \in H_{0}^{1}(\Omega)$ such that

$$
\sup _{t \geq 0} I_{\alpha}\left(t u_{1}\right)<\Lambda-D \lambda^{2 /(1+\gamma)},
$$

where $\Lambda$ and $D$ are as in Lemma 5.

Proof. Using $\lambda<(\Lambda / D)^{(1+\gamma) / 2}$, we have $\Lambda-D \lambda^{2 /(1+\gamma)}>0$ for any $\lambda \in\left(0,(\Lambda / D)^{(1+\gamma) / 2}\right)$. For $I_{\alpha}\left(t v_{\varepsilon}\right) \rightarrow \infty$, as $t \rightarrow \infty$, then there exists $t_{\varepsilon}>0$ such that $I_{\alpha}\left(t_{\varepsilon} v_{\varepsilon}\right)=\max _{t>0} I_{\alpha}\left(t v_{\varepsilon}\right)$. By Lemma 4, we obtain that $\max _{t>0} I_{\alpha}\left(t v_{\varepsilon}\right) \geq \rho>0$, and then $I_{\alpha}\left(t_{\varepsilon} v_{\varepsilon}\right) \geq \rho>0$. So, by the continuity of $I_{\alpha}$, we suppose that there exist positive constants $t_{0}, T_{0}$ such that $0<t_{0} \leq t_{\varepsilon} \leq T_{0}$. Set $I_{\alpha}\left(t_{\varepsilon} v_{\varepsilon}\right)=A\left(t_{\varepsilon} v_{\varepsilon}\right)+B\left(t_{\varepsilon} v_{\varepsilon}\right)$, where

$$
\begin{aligned}
A\left(t_{\varepsilon} v_{\varepsilon}\right)= & \frac{t_{\varepsilon}^{2}}{2} \int_{\Omega}\left(a\left|\nabla v_{\varepsilon}\right|^{2}+\left|v_{\varepsilon}\right|^{2}\right) d x \\
& +\frac{b}{4} t_{\varepsilon}^{4}\left(\int_{\Omega}\left|\nabla v_{\varepsilon}\right|^{2} d x\right)^{2} \\
& -\frac{1}{6} t_{\varepsilon}^{6} \int_{\Omega} Q(x) d x, \\
B\left(t_{\varepsilon} v_{\varepsilon}\right)= & \frac{\lambda}{1-\gamma} \int_{\Omega} f(x)\left[\alpha^{1-\gamma}-\left(t_{\varepsilon} v_{\varepsilon}+\alpha\right)^{1-\gamma}\right] d x \\
& -\frac{\mu}{2} t_{\varepsilon}^{2} \int_{\Omega}|x|^{\alpha-2} v_{\varepsilon}^{2} d x .
\end{aligned}
$$

Then,

$$
B\left(t_{\varepsilon} v_{\varepsilon}\right) \leq-\frac{\mu}{2} t_{\varepsilon}^{2} \int_{\Omega}|x|^{\alpha-2} v_{\varepsilon}^{2} d x
$$

Note that

$$
\frac{\mu}{2} t_{\varepsilon}^{2} \int_{\Omega}|x|^{\alpha-2} v_{\varepsilon}^{2} d x=c_{2} \varepsilon \frac{\alpha}{2}, \quad c_{2}>0,
$$

where $c_{2}$ is independent of $\varepsilon, \lambda$. From the above information, there holds

$$
\begin{aligned}
\sup _{t \geq 0} I_{\alpha}\left(t v_{\varepsilon}\right) & \leq \Lambda+c_{1} \varepsilon^{1 / 2}+B\left(t_{\varepsilon} v_{\varepsilon}\right) \\
& \leq \Lambda+c_{1} \varepsilon^{1 / 2}-c_{2} \varepsilon^{\alpha / 2} .
\end{aligned}
$$

Set $\varepsilon=\lambda^{4 /(1+\gamma)}, \Lambda_{0}=\left(c_{2} /\left(c_{1}+D\right)\right)^{(1+\gamma) / 2(1-\alpha)}$; when $\lambda \epsilon$ $\left(0, \Lambda_{0}\right)$, it follows that

$$
\sup _{t \geq 0} I_{\alpha}\left(t v_{\varepsilon}\right) \leq \Lambda-D \lambda^{2 /(1+\gamma)} .
$$

This completes the proof of Lemma 6.

Theorem 7. Since $\alpha \in(0,1)$, there exists $\lambda_{*}>0$ such that $\lambda \in\left(0, \lambda_{*}\right)$, and problem (12) has at least a positive solution $v_{\alpha} \in H_{0}^{1}(\Omega)$ such that $I_{\alpha}\left(v_{\alpha}\right)>\rho$, where $\rho$ is as in Lemma 2.

Proof. Set $\lambda_{*}=\min \left\{\lambda_{0}, \overline{\lambda_{0}}\right\}$; then, Lemmas 4-6 hold for $0<\lambda<\lambda_{*}$. From Lemma $4, I_{\alpha}$ satisfies the geometry of the mountain-pass lemma. Using the mountain-pass lemma, there exists a sequence $\left\{v_{n}\right\} \subset H_{0}^{1}(\Omega)$, such that

$$
\begin{aligned}
& I_{\alpha}\left(v_{n}\right) \longrightarrow c_{\alpha}>\rho, \\
& I_{\alpha}^{\prime}\left(v_{n}\right) \longrightarrow 0,
\end{aligned}
$$

where

$$
\begin{aligned}
c_{\alpha} & =\inf _{\gamma \in \Gamma \in[0,1]} \max _{\alpha}(\gamma(t)), \\
\Gamma & =\left\{\gamma \in C\left([0,1], H_{0}^{1}(\Omega)\right): \gamma(0)=0, \gamma(1)=u_{1}\right\} .
\end{aligned}
$$

Furthermore, by Lemmas 4 and 6, one has

$$
\begin{aligned}
0 & <\rho<c_{\alpha} \leq \max _{t \in[0,1]} I_{\alpha}\left(t u_{1}\right) \leq \sup _{t \geq 0} I_{\alpha}\left(t u_{1}\right) \\
& <\Lambda-D \lambda^{2 /(1+\gamma)} .
\end{aligned}
$$

Using Lemma 5, we obtain that $\left\{v_{n}\right\} \subset H_{0}^{1}(\Omega)$ has a convergent subsequence, still denoted by $\left\{v_{n}\right\}$, and we may suppose that $v_{n} \rightarrow v_{\alpha}$ in $H_{0}^{1}(\Omega)$ as $n \rightarrow \infty$. Then, from (74) and (72), we get

$$
I_{\alpha}\left(v_{\alpha}\right)=\lim _{n \rightarrow \infty} I_{\alpha}\left(v_{n}\right)=c_{\alpha}>\rho>0 .
$$

Therefore, by (73), we deduce that $v_{\alpha} \neq 0$. Hence, by the continuity of $I_{\alpha}^{\prime}$, we know that $v_{\alpha}$ is a solution of (12); namely,

$$
\begin{aligned}
\int_{\Omega}( & \left.a \nabla v_{\alpha} \nabla \varphi+v_{\alpha} \varphi\right) d x \\
& +b \int_{\Omega}\left|\nabla v_{\alpha}\right|^{2} d x \int_{\Omega} \nabla v_{\alpha} \nabla \varphi d x \\
& -\mu \int_{\Omega}|x|^{\alpha-2} v_{\alpha} \varphi d x-\int_{\Omega} Q(x)\left(v_{\alpha}^{+}\right)^{5} \varphi d x \\
& -\lambda \int_{\Omega} f(x) \frac{\varphi}{\left(v_{\alpha}^{+}\right)^{\gamma}} d x=0
\end{aligned}
$$


for all $\varphi \in H_{0}^{1}(\Omega)$. We can take the test function $\varphi=v_{\alpha}^{-}$in (74); one gets $\int_{\Omega}\left|\nabla v_{\alpha}^{-}\right| d x=0$, so $v_{\alpha}>0$ and $v_{\alpha} \neq 0$. From the strong maximum principle, we have that $v_{\alpha}$ is a positive solution of (12). The proof is completed.

\section{Existence of a Second Solution of Problem (1)}

In this section, we obtained a second solution to problem (1) as a limit point of a family of solutions $\left\{v_{\alpha}\right\}$ of (12).

Theorem 8. Assume $\lambda \in\left(0, \lambda_{*}\right)$, where $\lambda_{*}$ is as in Theorem 7 ; problem (1) admits a positive solution $v_{*}$ such that $I_{0}\left(v_{0}\right)>0$.

Proof. Set $\left\{v_{\alpha}\right\}$ as a family of positive solutions of (12); we can deduce that

$$
\begin{aligned}
& -\left(a+b \int_{\Omega}\left|\nabla v_{\alpha}\right|^{2} d x\right) \Delta v_{\alpha}+v_{\alpha} \\
& =Q(x) v_{\alpha}^{5}+\mu|x|^{\alpha-2} v_{\alpha}+\frac{\lambda f(x)}{\left(v_{\alpha}+\alpha\right)^{\gamma}} \\
& \quad \geq \min \left\{1, Q_{m}\right\} \min \left\{1, \frac{\lambda f_{m}}{2^{\gamma}}\right\},
\end{aligned}
$$

where $Q_{m}=\min Q(x), f_{m}=\min f(x)$, and then we have

$$
-\Delta v_{\alpha} \geq \frac{\min \left\{1, Q_{m}\right\} \min \left\{1, \lambda f_{m} / 2^{\gamma}\right\}-v_{\alpha}}{a+b \int_{\Omega}\left|\nabla v_{\alpha}\right|^{2} d x} .
$$

Noticing that $e$ is a weak solution of the problem

$$
\begin{aligned}
-\Delta u=1 & \text { in } \Omega, \\
u=0 & \text { on } \partial \Omega,
\end{aligned}
$$

hence it follows from the strong maximum principle that $e>$ 0 in $\Omega$, and for every $\Omega_{0} \subset \subset \Omega$, there exists $e_{0}>0$ such that $\left.e\right|_{\Omega_{0}} \geq e_{0}$. Then, from the comparison principle, we obtain

$$
v_{\alpha} \geq \frac{\min \left\{1, Q_{m}\right\} \min \left\{1, \lambda f_{m} / 2^{\gamma}\right\} e}{a+b \int_{\Omega}\left|\nabla v_{\alpha}\right|^{2} d x+e} .
$$

In particular, from $\left.e\right|_{\Omega_{0}} \geq e_{0}>0$, we get

$$
\left.v_{\alpha}\right|_{\Omega_{0}} \geq \frac{\min \left\{1, Q_{m}\right\} \min \left\{1, \lambda f_{m} / 2^{\gamma}\right\} e_{0}}{a+b \int_{\Omega}\left|\nabla v_{\alpha}\right|^{2} d x+e_{0}}>0 .
$$

Furthermore, from (72), (43), and (15), we have

$$
\begin{aligned}
\Lambda- & D \lambda^{2 /(1+\gamma)} \geq I_{\alpha}\left(v_{\alpha}\right)-\frac{1}{6}\left\langle I_{\alpha}^{\prime}\left(v_{\alpha}\right), v_{\alpha}\right\rangle \\
= & \frac{1}{3} \int_{\Omega}\left(a\left|\nabla v_{\alpha}\right|^{2}+\left|v_{\alpha}\right|^{2}\right) d x \\
& +\frac{b}{12}\left(\int_{\Omega}\left|\nabla v_{\alpha}\right|^{2} d x\right)^{2}-\frac{\mu}{3} \int_{\Omega}|x|^{\alpha-2}\left|v_{\alpha}\right|^{2} d x
\end{aligned}
$$

$$
\begin{aligned}
& -\frac{\lambda}{1-\gamma} \int_{\Omega} f(x)\left[\left(v_{\alpha}^{+}+\alpha\right)^{1-\gamma}-\alpha^{1-\gamma}\right] d x \\
& +\frac{\lambda}{6} \int_{\Omega} f(x) \frac{v_{\alpha}}{\left(v_{\alpha}^{+}+\alpha\right)^{\gamma}} d x \\
\geq & \frac{a}{3} \int_{\Omega}\left|\nabla v_{\alpha}\right|^{2} d x+\frac{b}{12}\left(\int_{\Omega}\left|\nabla v_{\alpha}\right|^{2} d x\right)^{2} \\
& -\frac{\mu}{3} \int_{\Omega}|x|^{\alpha-2}\left|v_{\alpha}\right|^{2} d x \\
& -\frac{\lambda}{1-\gamma} \int_{\Omega} f(x)\left(v_{\alpha}^{+}\right)^{1-\gamma} d x \\
\geq & \frac{1}{3}\left(a-\frac{\mu}{\mu_{1}}\right) \int_{\Omega}\left|\nabla v_{\alpha}\right|^{2} d x+\frac{b}{12}\left(\int_{\Omega}\left|\nabla v_{\alpha}\right|^{2} d x\right)^{2} \\
& -\frac{\lambda}{1-\gamma}|\Omega|^{(5+\gamma) / 6} S^{-(1-\gamma) / 2} \int_{\Omega} f(x)\left|\nabla u_{\alpha}^{+}\right|^{1-\gamma} d x .
\end{aligned}
$$

Therefore, $\left\{v_{\alpha}\right\}$ is bounded in $H_{0}^{1}(\Omega)$, and thus there exists a subsequence, still denoted by $\left\{v_{\alpha}\right\}$. We suppose that there exists $v_{0} \in H_{0}^{1}(\Omega)$ such that

$$
\begin{aligned}
v_{\alpha} & \rightarrow v_{0}, \quad \text { weakly in } H_{0}^{1}(\Omega), \\
v_{\alpha} & \longrightarrow v_{0}, \quad \text { strongly in } L^{p}(\Omega) \quad(1 \leq p<6), \\
v_{\alpha}(x) & \longrightarrow v_{0}(x), \quad \text { a.e. in } \Omega .
\end{aligned}
$$

Currently, we shall prove that $v_{\alpha} \rightarrow v_{0}$ in $H_{0}^{1}(\Omega)$ as $\alpha \rightarrow 0$.

In fact, we let $\omega_{\alpha}=v_{\alpha}-v_{0}$; then, $\int_{\Omega}|\nabla \omega| d x \rightarrow 0$ as $\alpha \rightarrow 0$. Otherwise, there exists a subsequence (still denoted by $\omega_{\alpha}$ ) such that $\lim _{\alpha \rightarrow 0} \int_{\Omega}\left|\nabla \omega_{\alpha}\right| d x=l>0$. Notice that $0 \leq v_{\alpha} /\left(v_{\alpha}+\alpha\right)^{\gamma} \leq v_{\alpha}^{1-\gamma}$; then, by the Vitali convergence theorem, we have

$$
\lim _{\alpha \rightarrow 0} \int_{\Omega} f(x) \frac{v_{\alpha}}{\left(v_{\alpha}+\alpha\right)^{\gamma}} d x=\int_{\Omega} f(x) v_{0}^{1-\gamma} d x .
$$

Therefore, by $\left\langle I_{\alpha}^{\prime}\left(v_{\alpha}\right), v_{\alpha}\right\rangle=0$ and Brézis-Lieb Lemma, one has

$$
\begin{gathered}
\int_{\Omega}\left(a\left|\nabla \omega_{\alpha}\right|^{2}+\left|\omega_{\alpha}\right|^{2}\right) d x+\int_{\Omega}\left(a\left|\nabla v_{0}\right|^{2}+\left|v_{0}\right|^{2}\right) d x \\
+b\left(\int_{\Omega}\left|\nabla \omega_{\alpha}\right|^{2} d x\right)^{2}+b\left(\int_{\Omega}\left|\nabla v_{0}\right|^{2} d x\right)^{2} \\
+2 b \int_{\Omega}\left|\nabla \omega_{\alpha}\right|^{2} d x \int_{\Omega}\left|\nabla v_{0}\right|^{2} d x \\
-\mu \int_{\Omega}|x|^{\alpha-2}\left|\omega_{\alpha}\right|^{2} d x-\mu \int_{\Omega}|x|^{\alpha-2}\left|v_{0}\right|^{2} d x \\
-\int_{\Omega} Q(x)\left(\omega_{\alpha}\right)^{6} d x-\int_{\Omega} Q(x)\left(v_{0}\right)^{6} d x \\
-\lambda \int_{\Omega} f(x)\left(v_{0}\right)^{1-\gamma} d x=o(1) .
\end{gathered}
$$


For $v_{\alpha} \rightarrow v_{0}$ in $H_{0}^{1}(\Omega)$ as $\alpha \rightarrow 0$, we can take the test function $\varphi=\phi \in H_{0}^{1}(\Omega) \cap C_{0}(\Omega)$ (here $C_{0}(\Omega)$ is the subset of $C(\Omega)$ consisting of functions with compact support in $\Omega$ ); taking the limit $\alpha \rightarrow 0$, one has $v_{0}(x)>0$ (by (79)); namely,

$$
\begin{aligned}
\int_{\Omega}( & \left.a \nabla v_{0} \nabla \phi+v_{0} \phi\right) d x+b \int_{\Omega}\left|\nabla v_{0}\right|^{2} d x \int_{\Omega} \nabla v_{0} \nabla \phi d x \\
& +b l^{2} \int_{\Omega} \nabla v_{0} \nabla \phi d x \\
= & \mu \int_{\Omega}|x|^{\alpha-2} v_{0} \phi d x+\int_{\Omega} Q(x)\left(v_{0}\right)^{5} \phi d x \\
& +\lambda \int_{\Omega} f(x)\left(v_{0}\right)^{-\gamma} \phi d x .
\end{aligned}
$$

We can assume that (84) holds for any $\phi \in H_{0}^{1}(\Omega)$. Indeed, since $H_{0}^{1}(\Omega) \cap C_{0}(\Omega)$ is dense in $H_{0}^{1}(\Omega)$, for any $\phi \in H_{0}^{1}(\Omega)$, there exists a sequence $\left\{\phi_{n}\right\} \in H_{0}^{1}(\Omega) \cap C_{0}(\Omega)$ such that $\phi_{n} \rightarrow \phi$, as $n \rightarrow \infty$. Since $n, m \in N^{+}$sufficiently large, by (84) written for $\phi=\phi_{n}-\phi_{m}$, we have

$$
\begin{aligned}
& \int_{\Omega}\left(a \nabla v_{0} \nabla\left|\phi_{n}-\phi_{m}\right|+v_{0}\left|\phi_{n}-\phi_{m}\right|\right) d x \\
& \quad+b \int_{\Omega}\left|\nabla v_{0}\right|^{2} d x \int_{\Omega} \nabla v_{0} \nabla\left|\phi_{n}-\phi_{m}\right| d x \\
& \quad+b l^{2} \int_{\Omega} \nabla v_{0} \nabla\left|\phi_{n}-\phi_{m}\right| d x \\
& =\mu \int_{\Omega}|x|^{\alpha-2} v_{0}\left|\phi_{n}-\phi_{m}\right| d x \\
& \quad+\int_{\Omega} Q(x)\left(v_{0}\right)^{5}\left|\phi_{n}-\phi_{m}\right| d x \\
& \quad+\lambda \int_{\Omega} f(x) v_{0}^{-\gamma}\left|\phi_{n}-\phi_{m}\right| d x .
\end{aligned}
$$

On the one hand, for $\phi_{n} \rightarrow \phi$, by (85), we can deduce that $\left\{\phi_{n} / v_{0}^{\gamma}\right\}$ is a Cauchy sequence in $L^{1}(\Omega)$, and thus there exists $\eta \in L^{1}(\Omega)$ such that $\phi_{n} / v_{0}^{\gamma} \rightarrow \eta(x)$, and then we get $\phi_{n} / v_{0}^{\gamma} \rightarrow \eta(x)$ in measure. From Riesz Theorem, $\left\{\phi_{n} / v_{0}^{\gamma}\right\}$ has a sequence, namely, $\left\{\phi_{n} / v_{0}^{\gamma}\right\}$, such that

$$
\frac{\phi_{n}}{v_{0}^{\gamma}} \longrightarrow \eta(x), \quad \text { a.e. } x \in \Omega \text {. }
$$

On the other hand, for $\phi_{n} / v_{0}^{\gamma} \rightarrow \phi / v_{0}^{\gamma}$ a.e. in $\Omega$, then, from (86), one has $\phi / v_{0}^{\gamma}=\eta(x)$. Therefore, $\int_{\Omega}\left(\phi_{n} / v_{0}^{\gamma}\right) d x \rightarrow$ $\int_{\Omega}\left(\phi / v_{0}^{\gamma}\right) d x$ as $n \rightarrow \infty$. Thus, we can take the test function $\phi=\phi_{n}$ in (84); taking the limit $n \rightarrow \infty$, hence we infer that (84) holds for $\phi \in H_{0}^{1}(\Omega)$.

In particular, we can take $\phi=v_{0}$ as the test function in (84); one has

$$
\begin{gathered}
\int_{\Omega}\left(a\left|\nabla v_{0}\right|^{2}+\left|v_{0}\right|^{2}\right) d x+b\left(\int_{\Omega}\left|\nabla v_{0}\right|^{2} d x\right)^{2} \\
+b l^{2} \int_{\Omega}\left|\nabla v_{0}\right|^{2} d x-\mu \int_{\Omega}|x|^{\alpha-2}\left(v_{0}\right)^{2} d x
\end{gathered}
$$

$$
\begin{aligned}
& -\int_{\Omega} Q(x)\left(v_{0}\right)^{6} d x-\lambda \int_{\Omega} f(x)\left(v_{0}\right)^{1-\gamma} d x \\
= & 0 .
\end{aligned}
$$

By (83) and (87), we deduce that

$$
\begin{aligned}
\int_{\Omega}( & \left.a\left|\nabla \omega_{\alpha}\right|^{2}+\left|\omega_{\alpha}\right|^{2}\right) d x+b\left(\int_{\Omega}\left|\nabla \omega_{\alpha}\right|^{2} d x\right)^{2} \\
& +b \int_{\Omega}\left|\nabla \omega_{\alpha}\right|^{2} d x \int_{\Omega}\left|\nabla v_{0}\right|^{2} d x \\
& -\mu \int_{\Omega}|x|^{\alpha-2}\left(\omega_{\alpha}\right)^{2} d x-\int_{\Omega} Q(x)\left(\omega_{\alpha}\right)^{6} d x \\
= & o(1) .
\end{aligned}
$$

It follows from (9) and (88) that

$$
\begin{aligned}
& \left(a-\frac{\mu}{\mu_{1}}\right) l^{2}+b l^{4}+b l^{2} \int_{\Omega}\left|\nabla v_{0}\right|^{2} d x \\
& \leq S^{-3} l^{6} \int_{\Omega} Q(x) d x,
\end{aligned}
$$

and then we get

$$
\geq \frac{b S^{3}+\sqrt{b^{2} S^{6}+4 S^{3} \int_{\Omega} Q(x) d x\left(a-\mu / \mu_{1}+b \int_{\Omega}\left|\nabla v_{0}\right|^{2} d x\right)}}{2 \int_{\Omega} Q(x) d x} .
$$

Furthermore, from Brézis-Lieb Lemma and (88), we obtain that

$$
\begin{aligned}
I\left(v_{0}\right)= & I_{\alpha}\left(v_{\alpha}\right) \\
& -\frac{1}{3} \int_{\Omega}\left[\left(a-\frac{\mu}{\mu_{1}}\right)\left|\nabla \omega_{\alpha}\right|^{2}+\left|\omega_{\alpha}\right|^{2}\right] d x \\
& -\frac{b}{12}\left(\int_{\Omega}\left|\nabla \omega_{\alpha}\right|^{2} d x\right)^{2}-\frac{b}{3} l^{2} \int_{\Omega}\left|\nabla \omega_{\alpha}\right|^{2} d x \\
& +o(1) .
\end{aligned}
$$
has

On the one hand, by (87), (15), and Young's inequality, one

$$
\begin{gathered}
I\left(v_{0}\right) \geq \frac{a}{3} \int_{\Omega}\left|\nabla v_{0}\right|^{2} d x-\frac{\mu}{3} \int_{\Omega}|x|^{\alpha-2}\left|v_{0}\right|^{2} d x-\frac{\lambda}{1-\gamma} \\
\cdot \int_{\Omega} f(x)\left(v_{0}\right)^{1-\gamma} d x-\frac{b}{4} l^{2} \int_{\Omega}\left|\nabla v_{0}\right|^{2} d x \geq \frac{1}{3}(a \\
\left.-\frac{\mu}{\mu_{1}}\right) \int_{\Omega}\left|\nabla v_{0}\right|^{2} d x-\frac{\lambda}{1-\gamma}|\Omega|^{(5+\gamma) / 6} \\
\cdot S^{-(1-\gamma) / 2} \int_{\Omega} f(x)\left|\nabla v_{0}\right|^{1-\gamma} d x-\frac{b}{4} l^{2} \int_{\Omega}\left|\nabla v_{0}\right|^{2} d x \\
\geq \frac{\mu(a-1)}{3 \mu_{1}} \int_{\Omega}\left|\nabla v_{0}\right|^{2} d x
\end{gathered}
$$




$$
\begin{array}{ll}
-\frac{1-\gamma}{2}\left[\left(\frac{2\left(a \mu_{1}-\mu\right)}{3 \mu_{1}(1-\gamma)}\right)^{(1-\gamma) / 2}\right. & -\frac{b}{4} l^{2} \int_{\Omega}\left|\nabla v_{0}\right|^{2} d x=-D \lambda^{2 /(1+\gamma)}-\frac{b}{4} \\
\left.\cdot \int_{\Omega}\left|\nabla v_{0}\right|^{1-\gamma} d x\right]^{2 /(1-\gamma)} & \cdot l^{2} \int_{\Omega}\left|\nabla v_{0}\right|^{2} d x .
\end{array}
$$

$$
-\frac{1+\gamma}{2}\left[\frac{\lambda|\Omega|^{(5+\gamma) / 6}\left(3 \mu_{1}(1-\gamma)\right)^{(1-\gamma) / 2} f_{m}}{\left(2\left(a \mu_{1}-\mu\right) S\right)^{(1-\gamma) / 2}}\right]^{2 /(1+\gamma)}
$$

On the other hand, since $I_{\alpha}\left(v_{\alpha}\right)<\Lambda-D \lambda^{2 /(1+\gamma)}$, then from (91) and (90) and taking $\alpha \rightarrow 0$, we obtained that

$$
\begin{aligned}
I_{\alpha}\left(v_{*}\right) \leq & \Lambda-D \lambda^{2 /(1+\gamma)}-\frac{1}{3}\left(a-\frac{\mu}{\mu_{1}}\right) l^{2}-\frac{1}{12} b l^{4}-\frac{1}{3} b l^{2} \int_{\Omega}\left|\nabla v_{*}\right|^{2} d x \\
\leq & \Lambda-D \lambda^{2 /(1+\gamma)}-\frac{1}{3}\left(a-\frac{\mu}{\mu_{1}}\right) \frac{b S^{3}+\sqrt{b^{2} S^{6}+4 S^{3} Q_{M}\left(a-\mu / \mu_{1}+b \int_{\Omega}\left|\nabla v_{*}\right|^{2} d x\right)}}{2 Q_{M}} \\
& -\frac{b}{12} \frac{b^{2} S^{6}+2 S^{3} Q_{M}\left(a-\mu / \mu_{1}+b \int_{\Omega}\left|\nabla v_{*}\right|^{2} d x\right)+b S^{4} \sqrt{b^{2} S^{4}+4 S Q_{M}\left(a-\mu / \mu_{1}+b \int_{\Omega}\left|\nabla v_{*}\right|^{2} d x\right)}}{2\left(Q_{M}\right)^{2}} \\
& -\frac{b}{3} \int_{\Omega}\left|\nabla v_{*}\right|^{2} d x \frac{b S^{3}+\sqrt{b^{2} S^{6}+4 S^{3} Q_{M}\left(a-\mu / \mu_{1}+b \int_{\Omega}\left|\nabla v_{*}\right|^{2} d x\right)}}{2 Q_{M}} \\
\leq & \Lambda-D \lambda^{2 /(1+\gamma)}-\frac{a b S^{3}}{6 Q_{M}}-\frac{b^{3} S^{6}}{24 Q_{M}^{2}}-\frac{a S \sqrt{b^{2} S^{4}+4 S^{3} Q_{M}\left(a-\mu / \mu_{1}+b \int_{\Omega}\left|\nabla v_{*}\right|^{2} d x\right)}}{6 Q_{M}} \\
& -\frac{b^{2} S^{4} \sqrt{b^{2} S^{4}+}+4 S^{3} Q_{M}\left(a-\mu / \mu_{1}+b \int_{\Omega}\left|\nabla v_{*}\right|^{2} d x\right)}{24 Q_{M}^{2}} \\
\leq & \Lambda-D \lambda^{2 /(1+\gamma)}-\Lambda-\frac{b}{4} l^{2} \int_{\Omega}^{\left|\nabla v_{*}\right|^{2} d x<-D \lambda^{2 /(1+\gamma)}-\frac{b}{4} l^{2} \int_{\Omega}\left|\nabla v_{*}\right|^{2} d x,} \\
& \Lambda \lambda^{2 /(1+\gamma)}-\frac{a b S^{3}}{6 Q_{M}}-\frac{b^{3} S^{6}}{24 Q_{M}^{2}}-\frac{a S \sqrt{b^{2} S^{4}+4 a S Q_{M}}}{6 Q_{M}}-\frac{b^{2} S^{4} \sqrt{b^{2} S^{4}+4 a S Q_{M}}}{24 Q_{M}^{2}}-\frac{b}{4} l_{\Omega}^{2}\left|\nabla v_{*}\right|^{2} d x
\end{aligned}
$$

which contradicts inequality (92). Hence, $v_{\alpha} \rightarrow v_{0}$ in $H_{0}^{1}(\Omega)$ as $\alpha \rightarrow 0$; that is, $l=0$; by (84), we deduce that $v_{0}$ is a positive solution of (1).

Finally, we only indicate that $I\left(v_{0}\right)>0$. For $v_{\alpha} \rightarrow v_{0}$ as $\alpha \rightarrow 0$, by Theorem 7, it follows that $I\left(v_{0}\right)=\lim _{\alpha \rightarrow 0} I_{\alpha}\left(v_{\alpha}\right)>$ $\rho>0$. This completes the proof of Theorem 8 .

\section{Conclusions}

In this paper, we studied the existence and multiplicity of positive solutions for the Schrödinger-Kirchhoff-type problem with singularity and critical exponents using the variational method, perturbation method, and the mountainpass lemma.

\section{Discussion}

In the future, a natural question is whether multiplicity of positive solutions for (1) can be established with the form of
Laplacian $\Delta_{p} u=\operatorname{div}\left(|\nabla u|^{p-2} \nabla u\right)$ (the $p$-Laplacian operator of $u, 1<p<3$ ). Another generalization is whether multiplicity of positive solutions for (1) can be established with multiple space dimensions in the form of $\Omega \subset \mathbb{R}^{N}(N>$ $3)$.

\section{Conflicts of Interest}

The authors declare that they have no conflicts of interest.

\section{Authors' Contributions}

All the authors contributed equally to this work, and all of them read and approved the final manuscript.

\section{Acknowledgments}

This research was supported by the National Natural Science Foundation of China (no. 11301489), the Distinguished Youth 
Science Foundation of Shanxi Province (2015021001), the Outstanding Youth Foundation of the North University of China (no. JQ201604), and the Youth Academic Leaders Support Program of the North University of China.

\section{References}

[1] G. B. Li and H. Y. Ye, "Existence of positive ground state solutions for the nonlinear Kirchhoff type equations in R3," Journal of Differential Equations, vol. 257, no. 2, pp. 566-600, 2014.

[2] S. Chen and X. Tang, "Infinitely many solutions for superquadratic Kirchhoff-type equations with sign-changing potential," Applied Mathematics Letters, vol. 67, pp. 40-45, 2017.

[3] N. B. Huy and B. T. Quan, "Positive solutions of logistic equations with dependence on gradient and nonhomogeneous Kirchhoff term," Journal of Mathematical Analysis and Applications, vol. 444, no. 1, pp. 95-109, 2016.

[4] G. M. Figueiredo and U. B. Severo, "Ground state solution for a Kirchhoff problem with exponential critical growth," Milan Journal of Mathematics, vol. 84, no. 1, pp. 23-39, 2016.

[5] B. Ricceri, "Energy functionals of Kirchhoff-type problems having multiple global minima," Nonlinear Analysis, vol. 115, pp. 130-136, 2015.

[6] C.-Y. Lei, J.-F. Liao, and C.-L. Tang, "Multiple positive solutions for Kirchhoff type of problems with singularity and critical exponents," Journal of Mathematical Analysis and Applications, vol. 421, no. 1, pp. 521-538, 2015.

[7] G. Kirchhoff, Mechanik, Teubner, Leipzig, 1883.

[8] J. L. Lions, "On some questions in boundary value problems of mathematical physics," North-Holland Mathematics Studies, vol. 30, no. C, pp. 284-346, 1978.

[9] X. He and W. Zou, "Existence and concentration behavior of positive solutions for a Kirchhoff equation in R3," Journal of Differential Equations, vol. 252, no. 2, pp. 1813-1834, 2012.

[10] P. D'Ancona and S. Spagnolo, "Global solvability for the degenerate Kirchhoff equation with real analytic data," Inventiones Mathematicae, vol. 108, no. 2, pp. 247-262, 1992.

[11] A. Arosio and S. Panizzi, "On the well-posedness of the Kirchhoff string," Transactions of the American Mathematical Society, vol. 348, no. 1, pp. 305-330, 1996.

[12] K. Perera and Z. Zhang, "Nontrivial solutions of Kirchhoff-type problems via the Yang index," Journal of Differential Equations, vol. 221, no. 1, pp. 246-255, 2006.

[13] M. Caponi and P. Pucci, "Existence theorems for entire solutions of stationary Kirchhoff fractional p-Laplacian equations," Annali di Matematica Pura ed Applicata, vol. 195, no. 6, pp. 2099-2129, 2016.

[14] G. Li and C. Yang, "The exietence of a nontrivial solution to a nonlinear elliptic boundary value problem of $p$-Laplacian type without the Ambrosetti-Rabinowitz condition," Nonlinear Analysis, vol. 72, no. 12, pp. 4602-4613, 2010.

[15] N. Lam and G. Lu, "Elliptic equations and systems with subcritical and critical exponential growth without the AmbrosettiRabinowitz condition," The Journal of Geometric Analysis, vol. 24, no. 1, pp. 118-143, 2014.

[16] N. Y. Lam and G. Z. Lu, "Elliptic equations and systems with subcritical and critical growth without the AmbrosettiRabinowitz condition," Advanced Nonlinear Studies, vol. 13, no. 2, pp. 289-308, 2013.
[17] P. K. Mishra and K. Sreenadh, "Fractional p-Kirchhoff system with sign changing nonlinearities," Reviata De La Real Academia De Ciencias Exactas Fisicas Y Naturales Serie A Matematicas, vol. 111, no. 1, pp. 281-296, 2017.

[18] J. Huang, C. Chen, and Z. Xiu, "Existence and multiplicity results for a $p$-Kirchhoff equation with a concave-convex term," Applied Mathematics Letters, vol. 26, no. 11, pp. 1070-1075, 2013.

[19] C.-Y. Lei, C.-M. Chu, H.-M. Suo, and C.-L. Tang, "On Kirchhoff type problems involving critical and singular nonlinearities," Annales Polonici Mathematici, vol. 114, no. 3, pp. 269-291, 2015.

[20] L. J. Shen and X. H. Yao, "Multiple positive solutions for a class of Kirchhoff type problems involving general critical growth," https://arxiv.org/abs/1607.01923.

[21] Y.-Y. Lan and C.-L. Tang, "Existence of solutions to a class of semilinear elliptic equations involving general subcritical growth," Proceedings of the Royal Society of Edinburgh, Section: A Mathematics, vol. 144, no. 4, pp. 809-818, 2014.

[22] C.-Y. Lei, H.-M. Suo, C.-M. Chu, and L.-T. Guo, "On ground state solutions for a Kirchhoff type equation with critical growth," Computers and Mathematics with Applications, vol. 72, no. 3, pp. 729-740, 2016.

[23] X. F. Cao, J. X. Xu, and J. Wang, "Multiple positive solutions for Kirchhoff type problems involving concave and critical nonlinearities in $R^{3}$," https://arxiv.org/abs/1605.06404.

[24] B. Cheng and X. Tang, "Ground state sign-changing solutions for asymptotically 3-linear Kirchhoff-type problems," Complex Variables and Elliptic Equations. An International Journal, vol. 62, no. 8, pp. 1093-1116, 2017.

[25] Q.-L. Xie, X.-P. Wu, and C.-L. Tang, "Existence and multiplicity of solutions for Kirchhoff type problem with critical exponent," Communications on Pure and Applied Analysis, vol. 12, no. 6, pp. 2773-2786, 2013. 


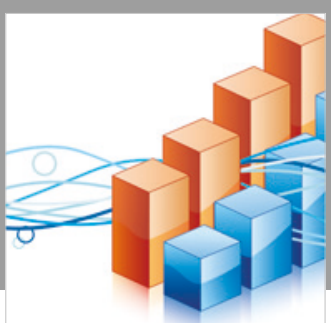

Advances in

Operations Research

\section{-n-m}
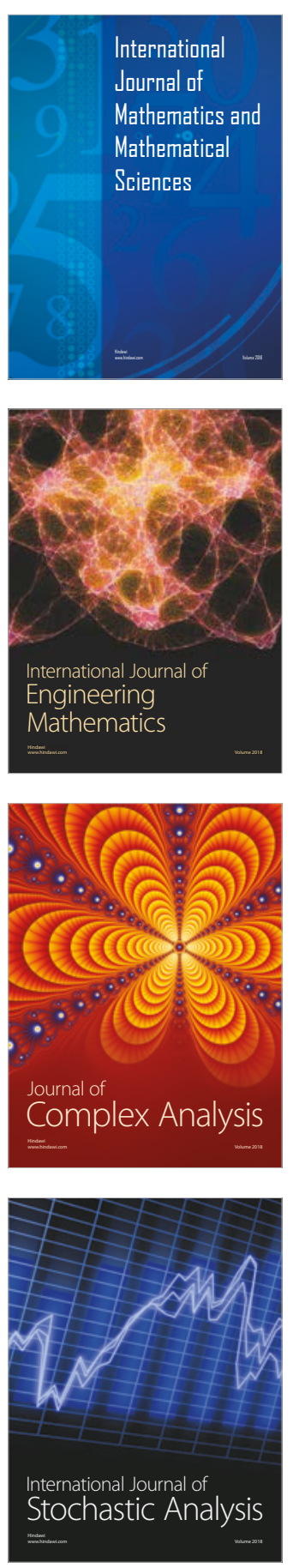
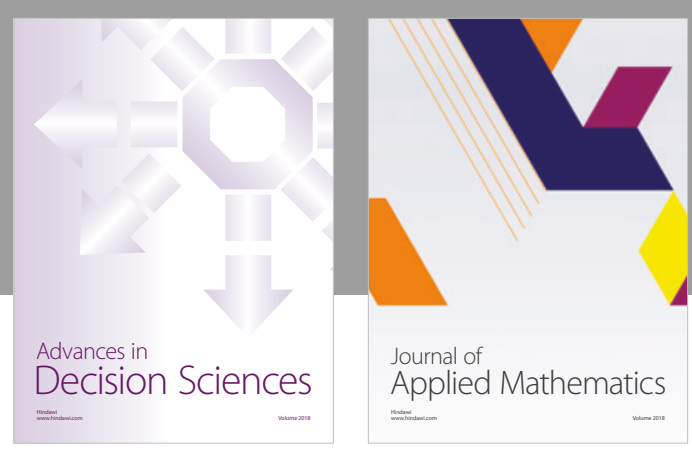

Journal of

Applied Mathematics
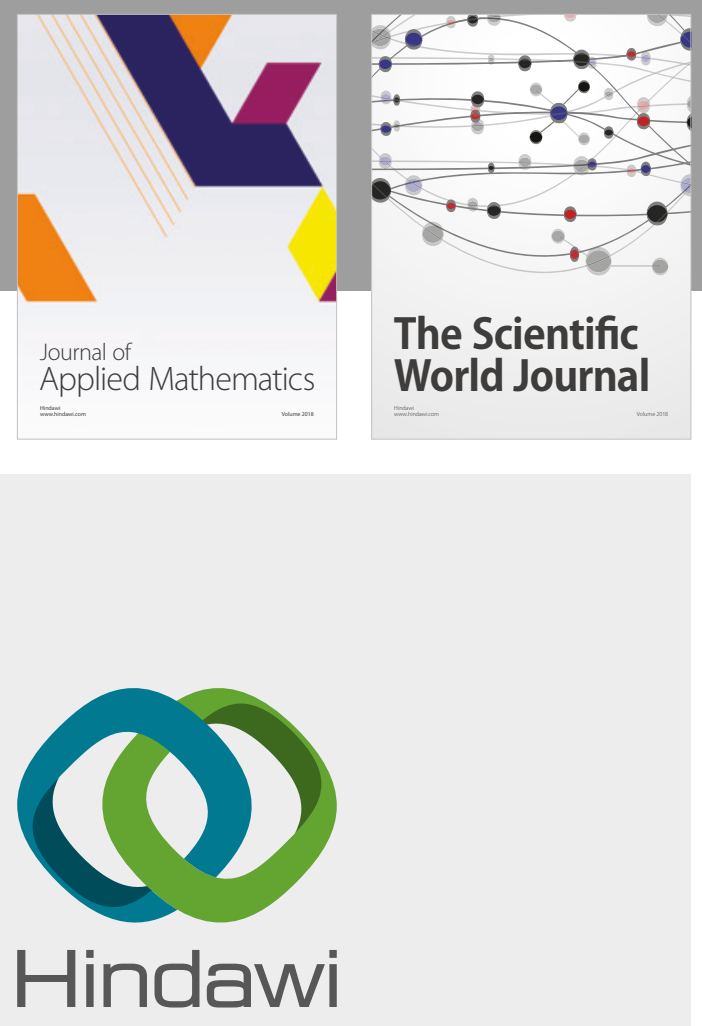

Submit your manuscripts at

www.hindawi.com

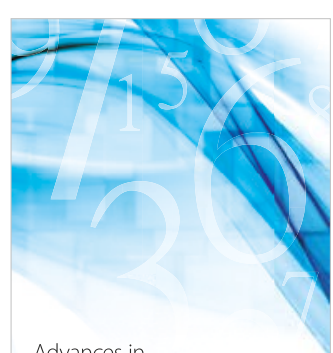

Advances in
Numerical Analysis
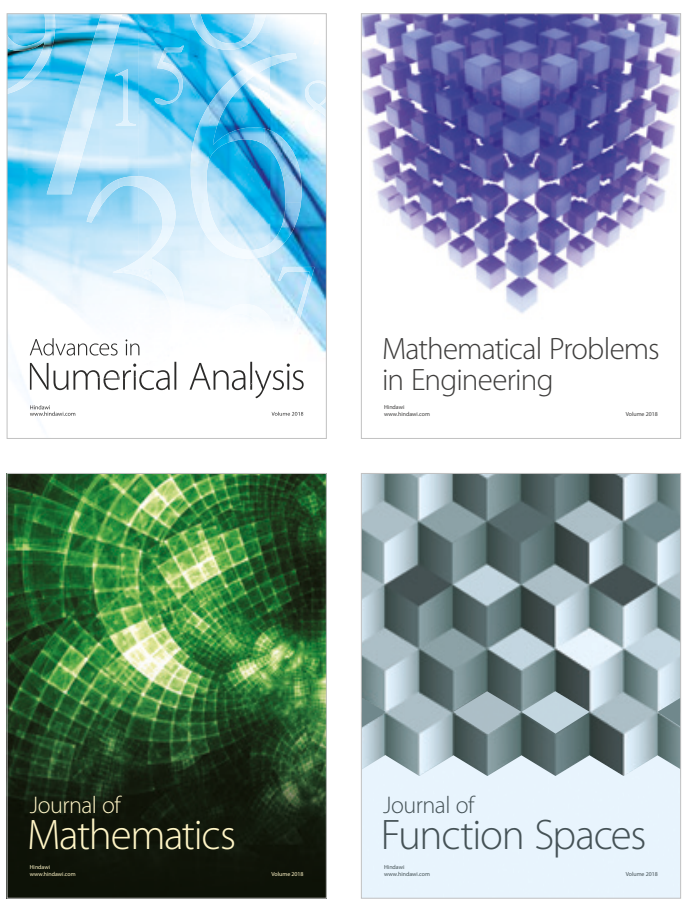

Mathematical Problems in Engineering

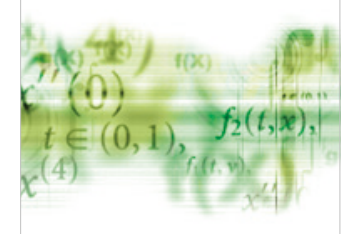

International Journal of

Differential Equations

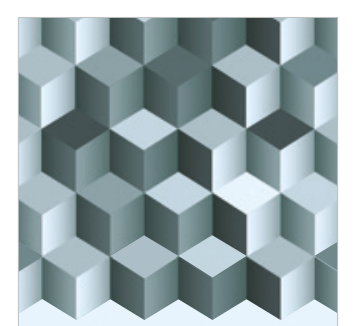

Journal of

Function Spaces

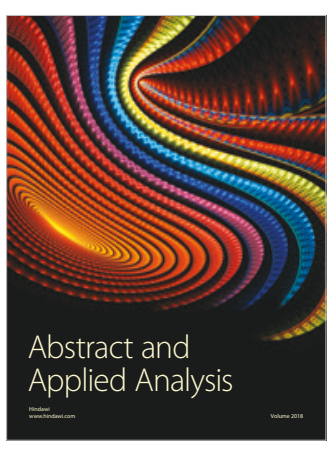

The Scientific

World Journal

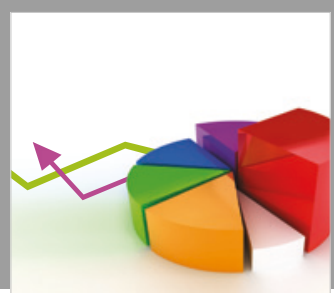

Journal of

Probability and Statistics
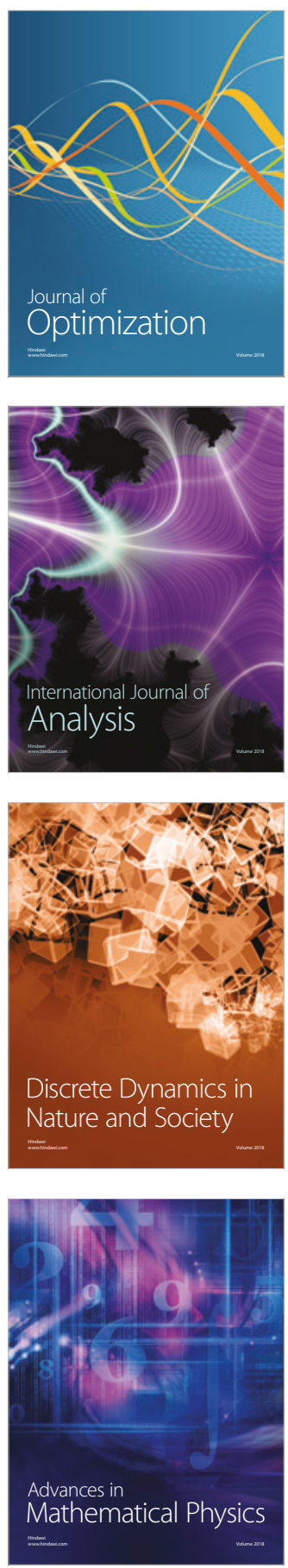